\title{
THE GLOBAL COSMOLOGICAL PARAMETERS
}

\author{
MASATAKA FUKUGITA \\ University of Tokyo, Institute for Cosmic Ray Research \\ Tanashi, Tokyo 188, Japan, and \\ Institute for Advanced Study, Princeton, NJ 08540, U. S. A.
}

\section{Introduction}

In these lectures I shall discuss the status of the determination of the three cosmological parameters which enter the Einstein equation and govern geometry and evolution of space-time of the Universe: the Hubble constant $H_{0}$, the mass density parameter $\Omega$ and the cosmological constant $\lambda$.

Among the three parameters, the Hubble constant is the dimensionfull quantity which sets the basic size and age of the Universe. The perennial effort to determine $H_{0}$ dates back to Hubble (1925) and has a long history of disconcordance. Recent progress has done much to resolve the long-standing discrepancy concerning the extragalactic distance scale, but there are some newly revealed uncertainties in the distance scale within the Milky Way. The emphasis in this lecture is on discussion of these uncertainties.

The mass density parameter directly determines the formation of cosmic structure. So, as our understanding of the cosmic structure formation is tightened, we should have a convergence of the $\Omega$ parameter. An important test is to examine whether the $\Omega$ parameter extracted from cosmic structure formation agrees with the value estimated in more direct ways. This gives an essential verification for the theory of structure formation.

The third important parameter in the Friedmann universe is the cosmological constant $\Lambda$. We now have some evidence for a non-zero $\Lambda$ which, if confirmed, would have most profound implications for fundamental physics. This lecture will focus on the strength of this 'evidence'.

We take the normalisation

$$
\Omega+\lambda=1
$$


for the flat curvature, where $\lambda=\Lambda / 3 H_{0}^{2}$ with $\Lambda$ the constant entering in the Einstein equation. The case with $\Omega=1$ and $\lambda=0$ is referred to as the Einstein-de Sitter (EdS) universe. We often use distance modulus

$$
m-M=5 \log \left(d_{L} / 10 \mathrm{pc}\right)
$$

instead of the distance $d_{L}$. For conciseness, we shall omit the units for the Hubble constant, $\left(\mathrm{km} \mathrm{s}^{-1} \mathrm{Mpc}^{-1}\right)$.

After the Summer Institute there appeared several important papers on the distance scale. I try to incorporate these results in this article.

\section{The Hubble Constant}

\subsection{HISTORICAL NOTE}

The global value of $H_{0}$ has long been uncertain by a factor of two. Before 1980 the dispute was basically between two schools: Sandage and collaborators had insisted on $H_{0}=50$ (Sandage \& Tammann 1982); de Vaucouleurs and collaborators preferred a high value $H_{0}=90-100$ (de Vaucouleurs 1981). Conspicuous progress was brought by the discovery of an empirical but tight relationship between galaxy's luminosity and rotation velocity, known as the Tully-Fisher relation (Tully \& Fisher 1977). The use of the Tully-Fisher relation has largely reduced subjective elements in the distance work, and $H_{0}=80-90$ has been derived from a straightforward reading of the Tully-Fisher relation. Representative of this work are the papers of Aaronson et al. (1986) and Pierce \& Tully (1988). A doubt was whether the result was marred with the Malmquist bias - whether the sample selects preferentially bright galaxies, and hence the result was biased towards a shorter distance (Kraan-Korteweg, Cameron \& Tammann 1988; Sandage 1993a). A related dispute was over the distance to the Virgo cluster, whether it is $16 \mathrm{Mpc}$ or $22 \mathrm{Mpc}$ : the different results depending on which sample one used.

The next momentous advancement was seen in 1989-1990 when a few qualified distance indicators were discovered. One of them is a technique using planetary nebula luminosity function (PNLF), the shape of which looked universal (Jacoby et al. 1990a). Another important technique is the use of surface brightness fluctuations (SBF), utilizing the fact that the images of distant galaxies show a smoother light distribution; while surface brightness does not depend on the distance, pixel-to-pixel fluctuations in a CCD camera decreases as $d_{L}^{-1}$ (Tonry \& Schneider 1988). They proposed that this smoothness can be a distance indicator if the stellar population is uniform. What was important is that the two completely independent methods predicted distances to individual galaxies in excellent agreement 
with each other (Ciardullo, Jacoby \& Tonry 1993). The PNLF/SBF distance also agreed with the value from the Tully-Fisher relation, with a somewhat larger scatter. These new techniques, when calibrated with the distance to M31, yielded a value around $H_{0}=80$ and the Virgo distance of $15 \mathrm{Mpc}$ (For a review of the methods, see Jacoby et al. 1992).

Around the same time the use of Type Ia supernovae (SNeIa) became popular (Tammann \& Leibundgut 1990; Leibundgut \& Pinto 1992; Branch \& Miller 1993). The principle is that the maximum brightness of SNIa is nearly constant, which can be used as an absolute standard candle. Arnett, Branch and Wheeler proposed that the maximum brightness is reliably calculable using models which are constrained from observations of released kinetic energy (Arnett, Branch \& Wheeler 1985; Branch 1992). This led to $H_{0}=50-55$, in agreement with the calibration based on the first Cepheid measurement of the nearest SNIa host galaxy using the pre-refurbished Hubble Space Telescope (HST) (Sandage et al. 1992). In the early nineties the discrepancy was dichotomous as whether $H_{0}=80$ or 50 . (see Fukugita, Hogan \& Peebles 1993 for the status at that time; see also van den Bergh 1989, 1994).

The next major advancement was brought with the refurbishment mission of HST, which enabled one to resolve Cepheids in galaxies as distant as $20 \mathrm{Mpc}$ (1994). This secured the distance to the Virgo cluster and tightened the calibrations of the extragalactic distance indicators, resulting in $H_{0}=(70-75) \pm 10,10 \%$ lower than the 'high value'. Another important contribution was the discovery that the maximum brightness of SNeIa varies from supernova to supernova, and that it correlates with the decline rate of brightness (Pskovskii 1984; Phillips 1993; Riess, Press \& Kirshner 1995; Hamuy et al. 1996a). This correction, combined with the direct calibration of the maximum brightness of several SNeIa with HST Cepheid observations, raised the 'low value' of $H_{0}$ to $65_{-10}^{+5}$, appreciably higher than 55 . This seemed to resolve the long-standing controversy.

All methods mentioned above use distance ladders and take the distance to Large Magellanic Clouds (LMC) to be $50 \mathrm{kpc}(m-M=18.5)$ as the zero point. Before 1997 few doubts were cast on the distance to LMC (TABLE 1 shows a summary of the distance to LMC known as of 1997). With the exception of RR Lyr, the distance converged to $m-M=18.5 \pm 0.1$, i.e., within 5\% error, and the discrepency of the RR Lyr distance was blamed on its larger calibration error. It had been believed that the Hipparcos mission (ESA 1997) would secure the distance within MW and tighten the distance to LMC. To our surprise, the work using the Hipparcos catalogue revealed the contrary; the distance to LMC was more uncertain than we had thought, introducing new difficulties into the determination of $H_{0}$. In this connection, the age of the Universe turned out to be more uncertain 
than it was believed.

During the nineties, efforts have also been conducted to determine the Hubble constant without resorting to astronomical ladders. They are called 'physical methods'. The advantage of the ladder is that the error of each ladder can be documented relatively easily, while the disadvantage is that these errors accumulate. Physical methods are free from the accumulation of errors, but on the other hand it is not easy to document the systematic errors. Therefore, the central problem is how to minimise the model dependence and document realistic systematic errors. Nearly ten years of effort has brought results that can be compared with the distances from ladders. The physical methods include the expansion photosphere model (EPM) for type II SNe (Schmidt, Kirshner \& Eastman 1992) and gravitational lensing time delay (Refsdal 1964). Use of SNeIa maximum brightness was once taken to be a physical method (Branch 1992), but then 'degraded' to be a ladder, which however significantly enhanced its accuracy.

TABLE 1. Distance to LMC as of 1997

\begin{tabular}{lll}
\hline Method & Ref & Distance moduli \\
\hline Cepheid optical PL & Feast \& Walker 1987 & $18.47 \pm 0.15$ \\
Cepheid optical PL & Madore \& Freedman 1991 & $18.50 \pm 0.10$ \\
Cepheid IR PL & Laney \& Stobie 1994 & $18.53 \pm 0.04$ \\
Mira PL & Feast \& Walker 1987 & $18.48 \pm(0.06)$ \\
SN1987A ring echo & Panagia et al. 1991 & $18.50 \pm 0.13$ \\
SN1987A EPM & Schmidt et al. 1992 & $18.45 \pm 0.13$ \\
RR Lyrae & van den Bergh 1995 & $18.23 \pm 0.04$ \\
\hline
\end{tabular}

\subsection{EXTRAGALACTIC DISTANCE SCALE}

The measurement of cosmological distances traditionally employs distance ladders (see Weinberg 1972). The most traditional ladders are shown in TABLE 2. The listings written in italic indicate new methods which circumvent intermediate rungs. The most important milestone of the ladder is LMC at 50kpc $(m-M=18.5)$. A distance indicator of particular historical importance (Hubble 1925) is the Cepheid period-luminosity (PL) relation, which is given a great confidence, but we note that it requires a few rungs of ladders to calibrate its zero point.

Prior to the HST work there were only $4-5$ galaxies with Cepheid distances which could be used to calibrate secondary indicators. The reach of the ground-based Cepheid measurement is about $3 \mathrm{Mpc}$, which means that 
TABLE 2. Traditional distance ladders

\begin{tabular}{lll}
\hline Method & Distance range & typical targets \\
\hline Population I stars & & \\
trigonometric or kinematic methods (ground) & $<50 \mathrm{pc}$ & Hyades, nearby dwarfs \\
main sequence fitting (FG stars) Pop. I & $<200 \mathrm{pc}$ & Pleiades \\
trigonometric method (Hipparcos) & $<500 \mathrm{pc}$ & nearby open clusters \\
main sequence fitting (B stars) & $40 \mathrm{pc}-10 \mathrm{kpc}$ & open clusters \\
Cepheids [Population I] (ground) & $1 \mathrm{kpc}-3 \mathrm{Mpc}$ & LMC, M31, M 81 \\
Cepheids [Population I] (HST) & $<30 \mathrm{Mpc}$ & Virgo included \\
secondary (extragalactic) indicators & $700 \mathrm{kpc}-100 \mathrm{Mpc}$ & \\
\hline Population II stars & & \\
trigonometric method (Hipparcos) & $<500 \mathrm{pc}$ & nearby subdwarfs \\
subdwarf main sequence fitting & $100 \mathrm{pc}-10 \mathrm{kpc}$ & global clusters \\
cluster RR Lyr & $5 \mathrm{kpc}-100 \mathrm{kpc}$ & LMC, age determinations \\
\hline
\end{tabular}

one cannot increase the number of calibrating galaxies from the ground. Pierce et al. (1994) could finally measure Cepheids in NGC 4571 in the Virgo cluster at $15 \mathrm{Mpc}$, but only with the best seeing conditions and difficult observations. The refurbishment of HST achieved a sufficient power to resolve Cepheids at the Virgo cluster (Freedman et al. 1994). Now 28 nearby spiral galaxies within $25 \mathrm{Mpc}$ are given distances measured using the Cepheid PL relation (Ferrarese et al. 1999b). A typical random error is $4-5 \%$ (0.08-0.10 mag), and the systematic error (from photometry) is $5 \%$ $(0.1 \mathrm{mag})$ excluding the uncertainty of the LMC distance, to which the HST-Key Project(KP) group assigns $6.5 \%$ error $(0.13 \mathrm{mag})$. The prime use of these galaxies is to calibrate secondary distance indicators which penetrate into a sufficient depth that perturbations in the Hubble flow are small enough compared with the flow itself.

Cepheids are Population I stars, so reside only in spiral galaxies. The calibration is therefore direct for TF and some SNeIa. For early type galaxies (fundamental plane or $D_{n}-\sigma$, and SBF) the calibration is not very tight; one must either use some groups where both early and late galaxies coexist, or regard the bulges of spiral galaxies as belonging to the same class as early galaxies and avoid contaminations from discs. Additional observations have been made for the galaxies that host SNeIa (Saha et al. 1999). The results are summarised in TABLE 3. We include a few earlier SNIa results which employ a partial list of Cepheid calibrators.

We accentuate the results with the two methods, SBF and SNeIa, in 
TABLE 3. Hubble constant

\begin{tabular}{lll}
\hline Secondary indicators & Refs & Hubble constant \\
\hline Tully-Fisher & HST-KP (Sakai et al. 1999) & $71 \pm 4 \pm 7$ \\
Fundamental Plane & HST-KP (Kelson et al. 1999) & $78 \pm 8 \pm 10$ \\
SBF & HST-KP (Ferrarese et al. 1999a) & $69 \pm 4 \pm 6$ \\
SBF & Tonry et al. (1999) & $\underline{77 \pm 4 \pm 7}$ \\
SNeIa & Riess et al. (1995) & $67 \pm 7$ \\
SNeIa & Hamuy et al. (1996b) & $63 \pm 3 \pm 3$ \\
SNeIa & Jha et al. (1999) & $64.4_{-5.1}^{+5.6}$ \\
SNeIa & Suntzeff et al. (1999) & $65.6 \pm 1.8$ \\
SNeIa & HST-KP (Gibson et al. 1999) & $\underline{68 \pm 2 \pm 5}$ \\
SNeIa & Saha et al. (1999) & $60 \pm 2$ \\
\hline Summary (see text) & & $(64-78) \pm 7$ \\
\hline
\end{tabular}

particular to those we underlined in the table. A cross correlation analysis showed that the relative distances agree well between SBF and others, including the Cepheid (Tonry et al. 1997; Freedman et al. 1997), and that it is probably the best secondary indicator presently available together with SNeIa; Also important is that there are now 300 galaxies measured with SBF, which are essential to make corrections for peculiar velocity flows for their $\leq 4000 \mathrm{~km} \mathrm{~s}^{-1}$ sample (Tonry et al. 1999). (PNLF is an indicator of comparable quality, but it requires more expensive observations so that applications are rather limited; see Jacoby et al. 1996 for the recent work.) The final value of Tonry et al. from their $I$ band survey is $H_{0}=77 \pm 8$, in which \pm 4 is allotted to uncertainties in the flow model and another \pm 4 to SBF calibration procedure in addition to the error of the Cepheid distance \pm 6 (a quadrature sum is taken). There are a several other pieces of the SBF work to determine $H_{0}$, which generally result in $H_{0}=70-90$ (e.g., Thomsen et al. 1997 using HST; Jensen et al. 1999 with $K$ band; see a review by Blakeslee et al. 1998). The new calibration made by the HST-KP group (Ferrarese et al. 1999a) would decrease $H_{0}$ only by $2 \%$. The difference in the final $H_{0}$ between Tonry et al. and Ferrarese et al. comes from using different targets (the latter authors use only 4 clusters) and flow models.

It is impressive that analyses of SNeIa Hubble diagram give virtually the same answer, even though the samples are all derived primarily from the Calán-Tololo sample of Hamuy et al. (1996b). A smaller $H_{0}$ of Saha et al. (1997) basically reflects the absence of the the luminosity-decline rate correction, which pushes up $H_{0}$ by $10 \%$. The other notable difference is a slightly higher value of HST-KP (Gibson et al. 1999), who made a reanalysis 
for all Cepheid observations performed by other groups and showed that their distances (to SN host galaxies) are all farther than would be derived from the HST-KP procedure. The average offset is as large as 0.16 mag $(8 \%)$. This correction applies to all results other than HST-KP should we keep uniformity of the Cepheid data reduction. This is important especially when one compares the SN results with those from other secondary distance indicators, since the calibrations for the latter exclusively use HST-KP photometry. Taking the luminosity-decline rate correlation to be real and adopting Cepheid distance from the HST-KP data reduction, I adopt $H_{0}=$ 68 from SNeIa.

We present two plots in Figure 1, (a) the estimates of maximum brightness of different authors and (b) the decline rate $\Delta m_{15}$, the amount of the decrease in brightness over 15 days following maximum light, both as a function of metallicity $[\mathrm{O} / \mathrm{H}]$. The second plot shows how metallicity effects are absorbed by the $M_{V}^{\max }-\Delta m_{15}$ relation and the first proves that there is little metallicity dependence in the corrected maximum brightness, though some scatter is seen among authors.

Leaving out the uncertainty of the Cepheid distance, $H_{0}$ from Tonry et al.'s SBF is $77 \pm 6$, and that from SNeIa (HST-KP) is $68 \pm 4$. The difference is $13 \%$, and the two values overlap at $H_{0}=71$. Allowing for individual two sigma errors, the overlap is in a range of $H_{0}=65-76$. An additional uncertainty is $6 \%$ error $\left(\delta H_{0}= \pm 4.5\right)$ from the Cepheid distance which is common to both, still excluding the uncertainty of the LMC distance. We may summarise $H_{0}=71 \pm 7$ or $64-78$ as our current standard, provided that LMC is at $50 \mathrm{kpc}$. All numbers in the table are within this range, except for the central value of Saha et al (1997).

In passing, let us note that $H_{0}=75 \pm 15$ (Freedman et al. 1997) obtained directly from the Cepheid galaxy sample agrees with the global value, implying that peculiar velocities are not so large even in a $10-20 \mathrm{Mpc}$ region.

This convergence is a great achievement, but keep in mind that the SNeIa results are still lower than those from other secondary indicators ${ }^{1}$ by $10 \%$. There are additional problems. First, all these analyses are based on a LMC distance modulus of $m-M=18.50$ (Feast \& Walker 1987; Madore \& Freedman 1991), which has recently been cast into doubt. In addition,

\footnotetext{
${ }^{1} \mathrm{~A}$ remark is given to the TF distance. While Sakai et al. (HST-KP) derived $H_{0}=71 \pm$ 8 using Giovanelli et al.'s (1997) cluster sample, Tammann and collaborators (Tammann 1999; Sandage \& Tammann 1997) insist on a low value $H_{0}=53-56$. Their cluster result (Federspiel et al. 1998) neglects the depth effect of the Virgo cluster: contrary to ellipticals, spiral galaxies are distributed elongating along the line of sight (Yasuda et al. 1996). Hence identifying the centre of gravity of the spiral galaxy distribution with the true core leads to an offset. In fact the presence of substructure behind the Virgo core is confirmed with the Cepheid for NGC4649. Tammann et al.'s field result comes from the allocation of an unusually large dispersion to the TF relation, which largely amplifies the Malmquist bias. Tully (1999) obtained $H_{0}=82 \pm 16$ (Tully et al. 1998).
} 

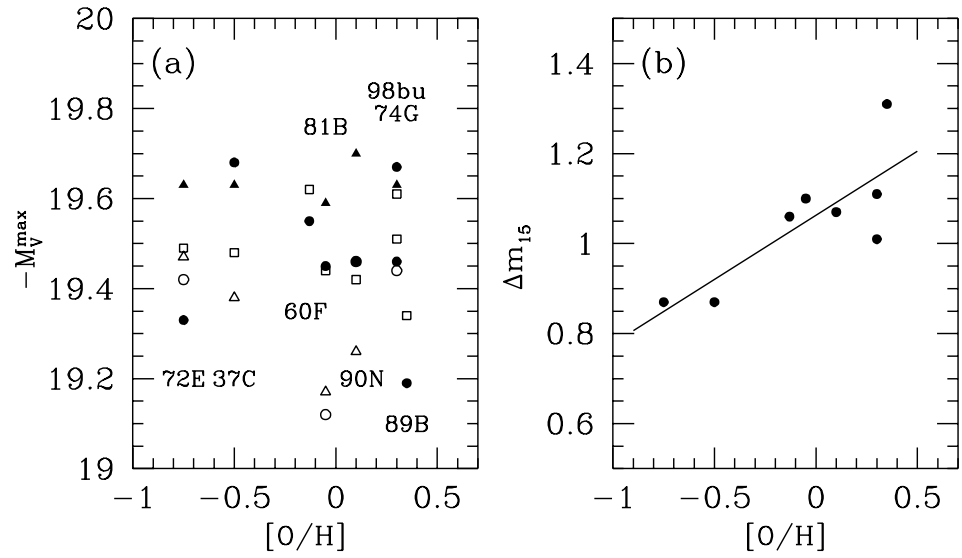

Figure 1. (a) Maximum brightness of SNeIa (in the $V$ band) adopted by different authors (see TABLE 3 ) as a function of $[\mathrm{O} / \mathrm{H}]$ of host galaxies: solid circles, Gibson et al. (1999); solid triangles, Suntzeff et al. 1999; open circles, Jha et al. (1999); open triangles, Hamuy et al. (1996b); open square, Saha et al. (1999). Note that Saha et al.'s calibration is not necessarily brighter, which is mainly due to a different treatment of extiction. (b) The decline rate $\Delta m_{15}$ measured in the $B$ band (Phillips et al. 1999) as a function of $[\mathrm{O} / \mathrm{H}]$ (Gibson et al. 1999). The slope of the curve is $\partial \Delta m_{15} / \partial[\mathrm{O} / \mathrm{H}] \simeq 0.28$.

metallicity effects could lead to systematic errors. Finally, should we derive the Hubble constant with the error of $10 \%$, the problem of dust extinction could be an issue, and it is potentially coupled with the metallicity. We now consider these issues in greater detail.

\subsection{DISTANCE TO LMC}

The present status of the LMC distance is given in TABLE 4. The most traditional paths to the LMC distance follow the ladder shown in the upper half of TABLE 2. The Hipparcos satellite can measure a parallax down to 2 milli arcsec (mas), corresponding to a distance of 500 pc (ESA 1997). It was a reasonable expectation that one could obtain the geometric distance to the Pleiades cluster, circumventing the main sequence fitting from nearby parallax stars to the Pleiades and thus securing the Galactic distance scale. Hipparcos observations have also opened a number of novel methods that can be used to estimate the distance to LMC. This and related activities, however, have actually brought confusions, rather than securing the distance scale within the MW. We discuss several issues in order. 
TABLE 4. Distance to LMC: Year 1997/1998

\begin{tabular}{lll}
\hline Method & Ref & Distance moduli \\
\hline Cepheid PL & Feast \& Catchpole 1997 & $18.70 \pm 0.10$ \\
& Paturel et al. 1997 & 18.7 \\
& Madore \& Freedman 1998 & $18.57 \pm 0.11$ \\
& Luri et al. 1998 & $18.29 \pm 0.17$ \\
& Luri et al. 1998 & $18.21 \pm 0.20$ \\
& (traditional) w/ new Pleiades & 18.26 \\
RR Lyrae (stat. para) & Fernley et al. 1998 & $18.31 \pm 0.10$ \\
& Luri et al. 1998 & $18.37 \pm 0.23$ \\
RR Lyrae (subdwarf) & Udalski 1998/Gould et al. 1998 & $18.09 \pm 0.16$ \\
Mira & Reid 1997 & 18.65 \\
Red clump & Gratton et al. 1997 & $18.60 \pm 0.07$ \\
& van Leeuwen et al. 1997 & $18.54 \pm 0.18$ \\
& Whitelock et al. 1997 & $18.60 \pm 0.18$ \\
& Udalski et al. 1998a & $18.08 \pm 0.15$ \\
Eclipsing binaries & Stanek et al. 1998 & $18.07 \pm 0.04$ \\
SN 1987A Ring echo & Cole 1998 & $18.36 \pm 0.17$ \\
& Guinan et al. 1998 & $18.30 \pm 0.07$ \\
& (Udalski et al. 1998b) & $18.19 \pm 0.13(?)$ \\
& Gould \& Uza 1998 & $<18.37 \pm 0.04$ \\
& Sonneborn et al. 1997 & $18.43 \pm 0.10$ \\
& Panagia et al. 1997 & $18.58 \pm 0.03$ \\
& Lundqvist \& Sonneborn1997 & $18.67 \pm 0.08$ \\
& Sekiguchi \& Fukugita 1998 & $18.10-18.60$ \\
& Sandage et al. 1999 & $18.57 \pm 0.05$ \\
& Gieren et al. 1997 & $18.49 \pm 0.05$ \\
\hline & & \\
& &
\end{tabular}

\subsection{1. "The Pleiades problem"}

The Pleiades cluster at $130 \mathrm{pc}$ has been taken to be the first milestone of the distance work, since it has nearly solar abundance of heavy elements. This cluster is already too far to obtain a reliable parallax with the ground based observations, and its distance is estimated by tying it with nearby stars with solar metallicity employing main sequence fitting of FGK dwarfs (e.g., van Leeuwen 1983). The distance obtained this way agrees with an estimate via the Hyades, the nearest cluster to which geometric distance is available from the ground (Hanson 1980; van Altena et al. 1997), after a correction for large metallicity of the Hyades (VandenBerg \& Bridges 1984). It was then a natural exercise to confirm these estimates with a parallax measured by the Hipparcos. The result showed that the Pleiades distance is 
shorter by 0.25 mag (12\%) (van Leeuwen \& Hansen-Ruiz 1997, Mermilliod et al 1997)! This is summarised in TABLE 5.

Mermilliod et al.'s (1997) (see also de Zeeuw et al. 1997) have shown that such a disagreement is seen not only for the Pleiades but also for other open clusters to some degree. A noteworthy example is that the locus of the Praesepe $([\mathrm{Fe} / \mathrm{H}]=+0.095)$ agrees with that of the Coma Ber $([\mathrm{Fe} / \mathrm{H}]=-0.065)$ without metallicity corrections, while we anticipate the former to be $0.25 \mathrm{mag}$ brighter due to higher metellicity.

This is a serious problem, since the disagreement means that either our understanding of FGK dwarfs, for which we have the best knowledge for stellar evolution, is incomplete, or the Hipparcos parallax contains systematic errors (Pinsonneault et al. 1998; Narayanan \& Gould 1999). The origin is not understood yet.

TABLE 5. Pleiades distance summary

\begin{tabular}{lll}
\hline Author/Method & Distance modulus \\
\hline van Leeuwen (1983) & $5.57 \pm 0.08$ \\
Lingå (1987) & 5.61 \\
\hline Hyades (Perryman et al. 1998) & $3.33 \pm 0.01$ & \\
Pleiades-Hyades & $2.52 \pm 0.05$ & \\
metallicity correction & $-0.22 \pm 0.03$ & \\
& & $5.63 \pm 0.06$ \\
\hline van Leeuwen \& Hansen-Ruiz 1997 & $5.32 \pm 0.05$ \\
Mermilliod et al. 1997 & $5.33 \pm 0.06$ \\
van Leeuwen 1999 & $5.37 \pm 0.07$ \\
\hline
\end{tabular}

\subsubsection{Metallicity effects in the LMC Cepheid calibration}

The Cepheid distance to LMC is based on the calibration using open cluster Cepheids, the distances to which are estimated by B star main sequence fitting that ties to the Pleiades (Sandage \& Tammann 1968, Caldwell, 1983, Feast \& Walker 1987, Laney \& Stobie 1994). Metallicity has been measured for some of these calibrator Cepheids (Fry \& Carney 1997). The residual of the PL fit shows a strong metallicity $(Z)$ dependence. This means either the Cepheid PL relation suffers from a large $Z$ effect, or the distances to open clusters contain significant $Z$-dependent errors (Sekiguchi \& Fukugita 1998). A correction for this effect changes the distance to LMC in either way, depending upon which interpretation is correct. 
This metallicity dependence problem can be avoided if parallaxes are used to find the distances to calibrator Cepheids. Attempts were made (Feast \& Catchpole 1997; Luri et al. 1998; Madore \& Freedman 1998) using field Cepheids in the Hipparcos catalogue. Unfortunately, Cepheid parallax data are so noisy (only 6 have errors less than 30\%) that they do not allow calibrations tighter than ladders. Another skepticism is that $2 / 3$ of Cepheids in the nearby sample (e.g., 14/26 in the Feast-Catchpole sample) are known to have companion stars, which would disturb the parallax (Szabados 1997).

\subsubsection{Red clump}

The OGLE group revived the use of the red clump (He burning stage of Population I stars) as a distance indicator. Paczyński \& Stanek (1998) showed that the $I$ band luminosity of the red clumps depends little on metallicity (see, Cole 1998, however), and gave a calibration using the Hipparcos parallax for nearby He burning stars. Udalski et al. (1998a) and Stanek et al. (1998) applied this to LMC, and obtained a distance modulus $18.1 \pm 0.1$, much shorter than those from other methods. This is a modern version of an analysis of Mateo \& Hodge (1986), who reported 18.1 \pm 0.3 . We should also recall that earlier analyses using MS fitting of OB stars resulted in a short distance of 18.2-18.3 (Schommer et al. 1984; Conti et al. 1986), though somewhat dismissed in the modern literature.

\subsubsection{Detached eclipsing binaries}

Detached double-spectroscopic eclipsing binaries provide us with a unique chance to obtain the distance in a semi-geometric way out to LMC or even farther. From the information given by the light curve and velocity curve, one can solve for the orbital parameters and stellar radii (Andersen 1991, Paczyński 1997; Bell et al 1993 for an earlier application to LMC HV2226; Torres et al. 1997 for an application to the Hyades). If surface brightness of the two stars is known from colour or spectrum, one can obtain the

distance as $d=(F / f)^{1 / 2} R_{i}$ where $F$ and $f$ are fluxes at the source and the observer and $R_{i}$ is stellar radius. Guinan et al. (1998) applied this method to HV2274 in LMC and derived $m-M=18.30 \pm 0.07$ with the aid of Kurucz' model atmosphere to estimate surface brightness from the spectrum. Udalski et al. (1998b) claimed that the extinction used is too small by an amount of $\Delta E(B-V)=0.037 \mathrm{mag}$ based on OGLE multicolour photometry. If we accept this correction the distance becomes 0.11 mag shorter, i.e., $m-M=18.19$. 


\subsubsection{RR Lyr problems}

In the first approximation the luminosity of RR Lyr is constant, but in reality it depends on metallicity. The dependence is usually expressed as

$$
\left\langle M_{V}(\mathrm{RR} \mathrm{Lyr})\right\rangle=a[\mathrm{Fe} / \mathrm{H}]+b .
$$

Much effort has been invested to determine $a$ and $b$. The problem is again how to estimate the distance to RR Lyr. Unlike the case with Cepheids, there are no unique ladders for the calibration, and a variety of methods have been used, of which the best known is the Baade-Wesselink method. The calibration from the ground may be summarised as

$$
\left\langle M_{V}(\mathrm{RR} \mathrm{Lyr})\right\rangle=0.2[\mathrm{Fe} / \mathrm{H}]+1.04 .
$$

With this calibration we are led to the LMC distance of $m-M \simeq 18.3$, as we saw in TABLE 1 above.

The Hipparcos catalogue contains a number of field subdwarfs with parallax. This makes a ladder available to calibrate RR Lyr in globular

clusters. Gratton et al. (1997) and Reid (1997) carried out this subdwarf fitting. Gratton et al. gave

$$
\left\langle M_{V}(\mathrm{RR} \text { Lyr })\right\rangle=(0.22 \pm 0.09)[\mathrm{Fe} / \mathrm{H}]+0.76 \text {. }
$$

Their data are plotted in Figure 2, together with (4) and (5). Reid's result is also consistent. This zero point, being brighter by $0.3 \mathrm{mag}($ at $[\mathrm{Fe} / \mathrm{H}]=-1.8)$ compared to (4), would bring the LMC distance to $m-M=18.5-18.6$.

There are a few analyses using the statistical parallax for field RR Lyr in the Hipparcos catalogue. Fernley et al. (1998) reported that their halo RR Lyr lie almost exactly on the curve of (4), rather than (5), and concluded a confirmation of the ground-based calibration. This is also endorsed by an analysis of Gould \& Popowski (1998).

The distance to eponymous RR Lyr was measured by Hipparcos. We see (Fig. 2) that RR Lyr itself does not fall on (5), but almost exactly on (4), although the error is fairly large. The uncertainties by 0.3 mag in the RR Lyr calibration translate to the LMC distance modulus 18.25-18.55.

\subsubsection{Conclusions on the LMC distance}

The distance to LMC is uncertain as much as $0.4 \mathrm{mag}$ ( $20 \%$ in distance), ranging from 18.20 to 18.60 . The results are rather bimodal around the two values close to the edges. A geometric method with SN1987A ring echo initiated by Panagia et al. 1991 does not differentiate between these two values: the data are too noisy and the result depends on the model of the light curve and emission lines that is adopted (Gould \& Uza 1997; Sonneborn et al. 1997; see Fig. 8 of the latter literature for the data quality). 


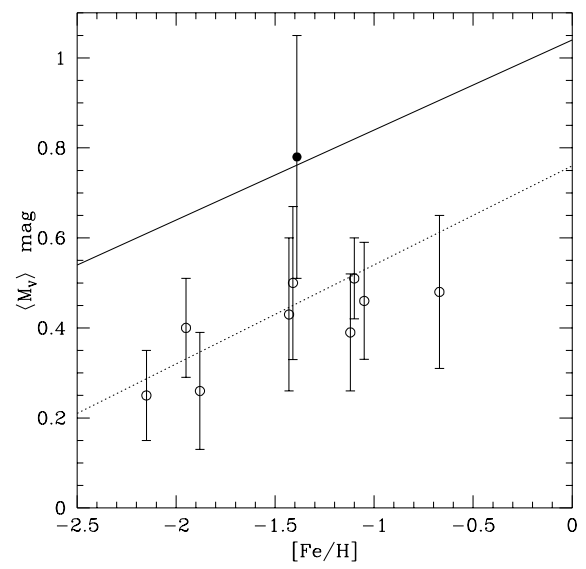

Figure 2. Calibrations of RR Lyr. The open points are taken from Gratton et al. (1997) with the dotted line indicating (5). The solid line is the ground-based calibration (4). The solid point denotes the eponymous RR Lyr measured by the Hipparcos satellite.

As we have seen in this section, recent observations with new techniques seem to tip the list to the lower value. This is clearly a systematic effect, so that we cannot simply take an 'average of all observations'. Rather, we should leave both possibilities open.

\subsubsection{Age of the globular clusters}

The RR Lyr calibration is also crucial in the estimation of the age of globular clusters, since the stellar age is proportional to the inverse of luminosity, i.e., inverse square of the distance. The modern evolution tracks of the main sequence agree reasonably well among authors. There are some disagreements in colours around the turn-off point, largely depending on the treatment of convection, but the luminosity is little affected (e.g., Renzini 1991; Vandenberg et al. 1996, especially their Fig. 1). Absolute magnitude at the turn-off point $M_{V}^{T O}$ of the main sequence is hence a good indicator of the age, as (Renzini 1991),

$$
\log t_{9}=-0.41+0.37 M_{V}^{T O}-0.43 Y-0.13[\mathrm{Fe} / \mathrm{H}],
$$

in units of Gyr, or

$$
\left.\log t_{9}=-0.41+(0.37 a-0.13)[\mathrm{Fe} / \mathrm{H}]+0.37\left[\left(M_{V}^{T O}-M_{V}^{\mathrm{RR}}\right)+b\right]\right]-0.43 Y,
$$

if (3) is included. The difference of the magnitudes between the turn-off point and $\mathrm{RR} \operatorname{Lyr}\left(M_{V}^{T O}-M_{V}^{\mathrm{RR}}\right)$ varies little among clusters and is measured to be $3.5 \pm 0.1 \mathrm{mag}$ (Buonanno et al. 1989; see Chaboyer et al. 1996 for a compilation). The metallicity dependence of the cluster age disappears 
if $a=0.35$, i.e., the globular cluster formation is coeval (Sandage 1993b). Both (4) and (5), however, give $a \simeq 0.2,{ }^{2}$ which indicates that metal-poor clusters appear older.

The dichotomous calibrations of RR Lyr obviously affect the age of globular clusters. Another large uncertainty is whether the age-metallicity correlation is real, indicating metal-poor clusters formed earlier, or is merely due to a systematic error, with the formation of globular cluster being coeval. The possibilities are four-fold:

$\begin{array}{cccc}b & (m-M)_{\text {LMC }} & t_{0} \text { (noncoeval) } & t_{0}(\text { coeval }) \\ 1.05 & 18.25 & 18 \mathrm{Gyr} & 15 \mathrm{Gyr} \\ 0.75 & 18.55 & 14 \mathrm{Gyr} & 12 \mathrm{Gyr}\end{array}$

In addition there are $\pm 10 \%$ errors from various sources (Renzini 1991; Bolte \& Hogan 1995; VandenBerg et al. 1996; Chaboyer et al. 1996). Figure 3 shows the age of various clusters from Gratton et al. (1997) and Chaboyer et al. (1998) both using the calibration close to (5). The $[\mathrm{Fe} / \mathrm{H}]$ dependence is apparent.

The claims of Gratton et al. (1997), Reid (1997) and Chaboyer et al. (1998) for young universe (11-12 Gyr) assume a coeval-formation interpretation together with the long RR Lyr calibration and take a mean of globular cluster ages. Three other possibilities, however, are not excluded.

\subsection{METALLICITY PROBLEMS WITH CEPHEIDS}

In most applications of the Cepheid PL relation, metallicity effects are neglected, motivated by theoretical arguments that they will be very small. This results from double cancellations of the metallicity dependences between core luminosity and atmosphere, as well as between the effects of the helium abundance and of heavier elements. The expected effect is (Stothers 1988, Iben\& Tuggle 1975; Chiosi et al. 1993)

$$
\gamma_{\lambda} \equiv \partial M_{\lambda} / \partial[\mathrm{Fe} / \mathrm{H}] \simeq \pm 0.05 \mathrm{dex}^{\mathrm{mag}^{-1}}
$$

for the $\lambda=V, I$ pass bands. A new calculation of Sandage et al. (1999) gives $\left|\gamma_{\lambda}\right|<0.1$ for $\lambda=B, V, I$.

When one is concerned with a $10 \%$ systematic error in the cosmic distance scale, the metallicity effect must be scrutinised. If it were as large as -0.5 , say, the true Cepheid distance to normal spiral galaxies would be

\footnotetext{
${ }^{2}$ A remark is made on a recent analysis of Kovács \& Jurcsik (1996), who obtained $a<0.19$ from a model-independent approach using the Fourier coefficients of the light curves that correlate with the metal abundance.
} 


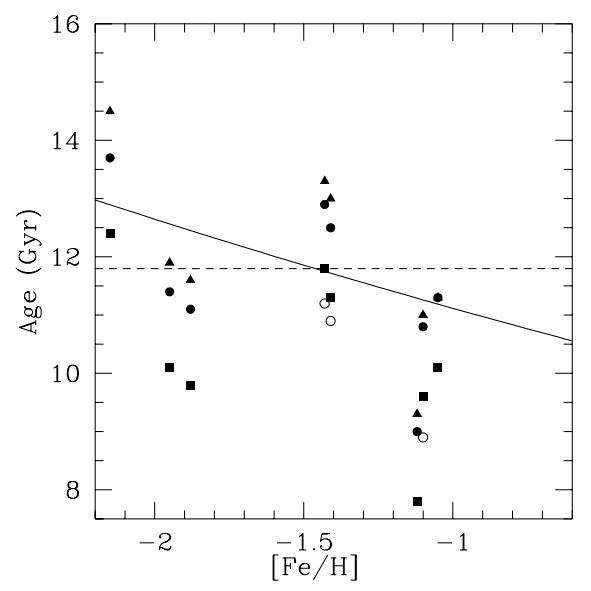

Figure 3. Age of globular clusters as a function of $[\mathrm{Fe} / \mathrm{H}]$. The solid points are from Gratton et al. (1997) for three different stellar evolution models. The open points are from Chaboyer et al. (1998). The solid line shows (7) but offset by -0.06 . The dashed line is 11.8 Gyr of Gratton et al.

longer by $10 \%$ relative to $\mathrm{LMC}([\mathrm{O} / \mathrm{H}]=-0.4)$. The calibrator $\mathrm{SNe}$ used in earlier papers (SN1937C, SN1972E, 1981B and 1990N) all reside in low $Z$ galaxies, but recent additions include SNe in high $Z$ galaxies (1989B, and notably $1998 \mathrm{bu}$ ), thus the sample spans a wider metallicity range (see Figure 1 above). There is now no relative difference in metallicity effects any more between the SBF and SNIa calibrator samples (the offset is $\Delta[\mathrm{O} / \mathrm{H}]<0.1)$. Therefore, we cannot ascribe the difference in $H_{0}$ to the metallicity effect of the Cepheid PL relation: the effect slightly reduces $H_{0}$ from both methods if the sign of $\gamma$ is negative.

However, it is important to know the magnitude of $\gamma$. Observationally, Freedman \& Madore (1990: FM) showed with the M31 data that the metallicity dependence is small $\left(\gamma_{B V R I}=-0.32 \pm 0.21\right)$. Gould (1994), however, reanalysed the same data and concluded it to be as large as $\gamma=-0.88 \pm 0.16$. The EROS collaboration derived $\gamma_{V I}=-0.44$ from a comparison between LMC and SMC (Beaulieu et al. 1997). Kochanek (1997) suggested $\gamma_{V I}=-0.14 \pm 0.14$ from a global fit of galaxies with Cepheid observations. The metallicity dependence for Galactic Cepheids discussed in section 2.3.2 corresponds to $\gamma_{V J H K} \approx-2$. Kennicutt et al. (1998) pointed out that the metallicity gradient of M31 used by Freedman \& Madore is a factor of three too large and argued that the above values should be $\gamma_{B V R I}=-0.94 \pm 0.78(\mathrm{FM})$ and $-2.1 \pm 1.1$ (Gould).

Kennicutt et al. (1998) derived from HST observations of two fields in M101 that $\gamma_{V I}=-0.24 \pm 0.16$, which is the value currently adopted in metallicity dependence analyses of the HST-KP group. If this is the true 
value, the effect on the distance scale is of the order of $5 \pm 3 \%\left(H_{0}\right.$ gets smaller). I would emphasize, however, that independent confirmations are necessary for this $\gamma$ value, since the M101 analysis is based only on $V$ and $I$ bands, and the effect of extinction might not be completely disentangled.

\subsection{CROSS-CHECK OF THE CEPHEID DISTANCES}

\subsubsection{Tests with geometric methods}

NGC4258 (M106) is a Seyfert 2 galaxy with $\mathrm{H}_{2} \mathrm{O}$ maser emission from clouds orbiting around a black hole of mass $4 \times 10^{7} M_{\odot}$ located at the centre. Precise VLBA measurements of Doppler velocities show that the motion of the clouds is very close to Keplerian and is perturbed very little (Miyoshi et al. 1995). A complete determination is made for the orbital parameters including centripetal acceleration and a bulk proper motion of the emission system. This yields a geometric distance to NGC4258 to be $7.2 \pm 0.3 \mathrm{Mpc}$ (Herrnstein et al. 1999).

Maoz et al. (1999) measured the distance to NGC4258 using the conventional Cepheid PL relation, and gave $8.1 \pm 0.4 \mathrm{Mpc}$ with $(m-M)_{\mathrm{LMC}}=18.5$. This distance is $13 \%$ longer than that from the maser measurement. The short LMC distance would bring the Cepheid distance in a perfect agreement with the geometric distance. This is, however, only one example, and it can merely be a statistical effect: the deviation is only twice the error, so it may happen with a chance probability of $5 \%$.

\subsubsection{Further checks for M31}

A number of distance estimates are available for the nearest giant spiral M31, and they are shown in TABLE 6 (the underlined numbers are the zero point). Stanek \& Garnavich (1998) applied the red clump method to M31, and obtained $m-M=24.47 \pm 0.06$, which agrees with the M31 modulus $24.44 \pm 0.10$ from the Cepheid based on the $(m-M)_{\mathrm{LMC}}=18.5$ calibration, whereas the same method gives 18.1 for the LMC distance. Namely, M31-LMC largely disagrees between the two. This discrepancy might be ascribed to a metallicity effect of either Cepheids or red clumps, or to the systematic error of either indicator. Mochejska et al. (1999) ascribed it to the error of the Cepheid distance from a crowded stellar population. On the other hand, tip of giant branch (TRGB) gives $(m-M)_{\mathrm{M} 31-\mathrm{LMC}}$ in agreement with the value derived from the Cepheid. The difference from PNLF is also consistent. The value from RR Lyr, however, is consistent with that from red clumps (the numbers in the Table is derived using (4) for the zero point). The results are dichotomous again. 
TABLE 6. Relative distance of M31 to LMC

\begin{tabular}{lllll}
\hline method & M31 & LMC & M31-LMC & refs. \\
\hline Cepheid & $24.44 \pm 0.10$ & $\underline{18.5}$ & $5.94 \pm 0.10$ & Ferrarese et al. 1999b \\
red clump & $24.47 \pm 0.06$ & $18.07 \pm 0.04$ & $6.40 \pm 0.07$ & Stanek \& Garnavich 1998 \\
TRGB & $24.41 \pm(0.19)$ & $\underline{18.5}$ & $5.91 \pm(0.19)$ & Ferrarese et al. 1999b \\
RR Lyr $(B)$ & $24.50 \pm(0.15)$ & 18.30 & $6.20 \pm$ & Pritchet \& vd Bergh 1989 \\
PNLF & $\underline{24.44}$ & $18.56 \pm 0.18$ & $5.82 \pm 0.18$ & Jacoby et al. 1990b \\
\hline
\end{tabular}

\subsection{PHYSICAL METHODS}

2.6.1. Expansion photosphere model (EPM) for Type II supernovae

This is a variant of the Baade-Wesselink method. If a supernova is a black body emitter one can calculate source brightness from temperature; the distance can then be estimated by comparing source brightness with the observed flux. In SNeII atmosphere the flux is diluted due to electron scattering opacity. If this greyness is calculated source brightness can be inferred. Schmidt, Kirshner \& Eastman (1992) developed this approach and obtained the distances to SNeII in agreement with those from the ladder. The point is that EPM gives absolute distance without zero point calibrations. The Hubble constant they obtained is $73 \pm 9$ (Schmidt et al. 1994).

A possible source of systematic errors is in the estimation of the temperature from the spectrum or colour. The SNeII physics also might not be uniform, as we see occasional large scatters in a cross-correlation analysis.

\subsubsection{Gravitational lensing time delay}

When quasar image is split into two or more by gravitational lensing, we expect the time delay among images, arising from different path lengths and gravitational potentials among image positions. The time delay between images $\mathrm{A}$ and $\mathrm{B}$ takes the form

$$
\Delta t=\frac{1+z_{L}}{H_{0}}\left(\frac{D_{O L} D_{O S}}{D_{L S}} H_{0}\right)\left[\frac{1}{2}\left(\left|\theta_{A}\right|^{2}-\left|\theta_{B}\right|^{2}-\left.\Delta \phi\right|_{A-B}\right)\right]
$$

where $\theta$ is the angular difference between the source and image, $\Delta \phi$ is the difference in the potential and $D_{I J}$ is the angular diameter distances. The time delay is observable if the source is variable, and can be used to infer $H_{0}$ (Refsdal 1964). Crucial in this argument is a proper modelling of the mass distribution of the deflector. The $D D / D$ factor depends on $\Omega$ only weakly; its $\lambda$ dependence is even weaker. 
The first case where $H_{0}$ is derived is with the $0957+561$ lens system. The deflector is complicated by the fact that a giant elliptical galaxy is embedded into a cluster. Falco, Gorenstein \& Shapiro (1991) noted an ambiguity associated with a galaxy mass - cluster mass separation, which does not change any observed lens properties but affects the derived Hubble constant. One way to resolve this degeneracy is to use the velocity dispersion of the central galaxy (Falco et al. 1991; Grogin \& Narayan 1996). Kundić et al. (1997b), having resolved a long-standing uncertainty about the time delay, obtained $H_{0}=64 \pm 13$ employing the Grogin-Narayan model. Tonry \& Franx (1998) revised it to $71 \pm 7$ with their new velocity dispersion measurement near the central galaxy. More recently, Bernstein \& Fischer (1999) searched a wider variety of models, also using weak lensing information to constrain the mass surface density of the cluster component, and concluded $H_{0}=77_{-24}^{+29}$, the large error representing uncertainties associated with the choice of models.

The second example, PG1115+080, is again an unfortunate case. The deflector is elliptical galaxy embedded in a Hickson-type compact group of galaxies (Kundić et al. 1997a). Keeton \& Kochanek (1997) and Courbin et al. (1997) derived $(51-53) \pm 15$ from the time delay measured by Schechter et al. (1997). Impey et al. (1998) examined the dependence of the derived $H_{0}$ on the assumption for the dark matter distribution, and found it to vary from $44 \pm 4$ (corresponding to $M / L$ linearly increasing with the distance) to $65 \pm 5$ (when $M / L$ is constant over a large scale). The latter situation may sound strange, but it seems not too unusual for elliptical galaxies, a typical example being seen in NGC5128 (Peng et al. 1998).

Recently, time delays have been measured for three more lenses, B0218+ 357, B1608+656 and PKS1830-211. B0218+357 is a rather clean, isolated spiral galaxy lens, and Biggs (1999) derived $H_{0}=69_{-19}^{+13}$ (the central value will be 74 if $\Omega=0.3$ ) with a simple galaxy model of a singular isothermal ellipsoid. For B1608+656, Koopmans \& Fassnacht (1999) obtained $64 \pm 7$ for $\Omega=0.3$ ( $59 \pm 7$ for EdS). For PKS1830-211, they gave $75_{-10}^{+18}$ for EdS and $85_{-11}^{+20}$ for $\Omega=0.3$ from the time delay measured by Lovell et al. (1998). More work is clearly needed to exhaust the class of models, but these three lens systems seem considerably simpler than the first two examples. Koopmans \& Fassnacht concluded $74 \pm 8$ for low density cosmologies $(69 \pm 7$ for EdS) from four (excluding the second) lensing systems using the simplest model of deflectors. It is encouraging to find a good agreement with the values from the ladder argument, though the current results from lenses are still less accurate than the ladder value. It would be important to ask whether $H_{0}<60$ or $>80$ is possible within a reasonable class of deflector models. 


\subsubsection{Zeldovich-Sunyaev effect}

The observation of the Zeldovich-Sunyaev (ZS) effect for clusters tells us about the cluster depth (times electron density), which, when combined with angular diameter (times electron density square) from X ray observations, gives us the distance to the cluster provided that cluster is spherical (Birkinshaw et al. 1991, Myers et al. 1997). This is often taken as a physical method to measure $H_{0}$. I give little weight to this method in these lectures, since it is difficult to estimate the systematic errors. The currently available results wildly vary from a cluster to a cluster. The most importnat is a bias towards elongation. None of the known clusters are quite spherical, and selection effects bias towards clusters elongated along the line of sight because of higher surface brightness. This may happen even if one uses a large sample. Additional systematics arise from the sensitivity of the ZS effect to the cluster envelopes; one must resort to a model to correct for this effect.

\subsubsection{Physical methods: summary}

Physical methods now yield the Hubble constant which can be compared with that from ladders. TABLE 7 presents a summary of $H_{0}$ from the physical methods. However, effort is still needed to determine systematic errors associated with the use of specific methods.

TABLE 7. Hubble constant from ladders and physical methods

\begin{tabular}{|c|c|c|}
\hline method & $H_{0}$ & reference \\
\hline ladders & $71 \pm 7(\times 0.95-1.15)$ & \\
\hline physical: EPM & $73 \pm 9$ & Schmidt et al. 1994 \\
\hline physical: lensing (low $\Omega$ ) & $74 \pm 8$ & Koopmans \& Fassnacht 1999 \\
\hline$(\mathrm{EdS})$ & $69 \pm 7$ & \\
\hline physical: ZS & $(54 \pm 14)$ & Myers et al 1997 \\
\hline
\end{tabular}

\subsection{CONCLUSIONS ON $H_{0}$}

The progress in determining the extragalactic distance scale has been dramatic. The ladders yield values convergent within $10 \%$, which is compared to a factor 1.6 disagreement in the early nineties. A new uncertainty, however, becomes manifest in the Galactic distance scale: there is a 15-20\% uncertainty in the distance to LMC. Therefore, we may summarise

$$
H_{0}=(71 \pm 7) \times \begin{aligned}
& 1.15 \\
& 0.95
\end{aligned}
$$


as a currently acceptable value of the Hubble constant. This agrees with that from HST-KP (Mould et al. 1999) up to the uncertainty from the LMC distance, though we followed a different path of argument. This allows $H_{0}=90$ at the high end (if Tonry et al's SBF is weighted) and 60 at the low end (if the SNeIa results are weighted). Note that $H_{0}$ from both EPM and gravitational lensing are consistent with the ladder value for $(m-M)_{\mathrm{LMC}}=$ 18.5. With the shorter LMC distance the overlap is marginal.

The short LMC distance will also cause trouble for the $H_{0}$-age consistency. The LMC distance modulus of $m-M=18.25$ would raise the lower limit of $H_{0}$ to 72 , and increase the lower limit of age from $\approx 11.5 \mathrm{Gyr}$ to $\approx 14.5 \mathrm{Gyr}$ at the same time. There is then no solution for a $\lambda=0$ universe. With a non-zero $\lambda$, a unique solution is $H_{0} \simeq 72, \Omega \simeq 0.25, \lambda \simeq 0.75$ with coeval globular cluster formation (see Figure 6 below).

In the future it is likely that more effort will be expended for geometric methods. The great advantage is that it is free from errors arising from the chemical composition. In the surface brightness method, the chemical composition may still enter into the game, but its effect is tolerable and can even be reduced to a negligible level by using near infrared observations.

Ultimately, gravitational wave observations could provide us with a novel method. For instance, for coalescing binary neutron stars the distance can be calculated as $d \sim \nu^{-2} \varepsilon^{-1} \tau^{-1}$, where $\varepsilon$ is metric perturbations, $\nu$ is the frequency and $\tau=\nu / \dot{\nu}$ is a characteristic time of the collapse (Schutz 1986). The position of the object may be difficult to infer, but there might be a gamma ray burst associated with the coalescence.

\section{The density parameter}

\subsection{MODEL-INDEPENDENT DETERMINATIONS}

\subsubsection{Luminosity density $\times\langle M / L\rangle$}

The mass density can be obtained by multiplying the luminosity density with galaxy's average mass to light ratio $\langle M / L\rangle$. The local luminosity density, evaluated by integrating the luminosity function, is reasonably well converged to $\mathcal{L}_{B}=(2.0 \pm 0.4) \times 10^{8} h L_{\odot} \mathrm{Mpc}^{-3}$ from many observations. The $M / L_{B}$ of galaxies generally increases with the scale. When the mass is integrated to $\approx 100 \mathrm{kpc}$, a typical $M / L_{B}$ is about $(100-200) h$ in solar units, and it may still increase outward (e.g., Faber \& Gallagher 1979; Little \& Tremaine 1987; Kochanek 1996; Bahcall et al. 1995; Zaritsky et al. 1997). The virial radius in a spherical collapse model

is $r=0.13 \mathrm{Mpc} \Omega^{-0.15}\left[M / 10^{12} M_{\odot}\right]_{<100 \mathrm{kpc}}^{1 / 2}$. If the dark matter distribution is isothermal within the virial radius, the value of $M / L_{B}$ inside the virial radius is $(150-400) h$ for $L^{*}$ galaxies. This is about the value of $M / L_{B}$ for groups and clusters, $(150-500) h$. Multiplying the two values we get 
$\Omega=0.20 \times 2^{ \pm 1}$. See also Fukugita, Hogan \& Peebles (1998) for variants of this argument.

Carlberg et al. $(1996,1997 \mathrm{a})$ tried to make the argument more quantitative using their cluster sample and a built-in field galaxy sample. They estimated $M / L_{r} \simeq(210 \pm 60) h$ for field galaxies from the cluster value $(289 \pm$ $50) h$. Their luminosity density of field galaxies is $\mathcal{L}_{r}=(1.7 \pm 0.2) \times 10^{8} h L_{\odot}$ $\mathrm{Mpc}^{-3}$, and therefore $\Omega_{0}=0.19 \pm 0.06$. Note that $M / L_{B} \simeq 1.4 \times M / L_{r}$ in solar units for the respective pass bands.

The important assumption for these calculations is the absence of copious matter outside the clusters. This is a question difficult to answer, but the observation of weak lensing around the clusters indicate that the distributions of dark mass and galaxies are similar at least in the vicinity of clusters (Tyson \& Fischer 1995; Squires et al. 1996).

Some attempts have also been made to estimate the mass on a supercluster scale. Small et al. (1998) inferred $M / L_{B} \simeq 560 h$ for the Corona Borearis supercluster, by applying the virial theorem (inspired by an $N$ body simulation). On the other hand, Kaiser et al. (1998) estimated $M / L_{B} \simeq 250$ from a mesurement of the gravitational shear of weak lensing caused by a supercluster MS0302 $+17^{3}$; the result is not well convergent, but it seems unlikely that $\Omega$ is larger than 0.5 .

\subsection{2. $H_{0}$ versus cosmic age}

For $H_{0} \geq 60$, the age is 10.9 Gyr for the EdS universe. This is too short. $\Omega$ must be smaller than unity. If we take $t_{0}>11.5$ Gyr $\Omega<0.7$. The limit is weak, but the significance is that EdS universe is nearly excluded.

\subsubsection{Type Ia supernova Hubble diagram}

The type Ia supernova Hubble diagram now reaches $z \simeq 0.4-0.8$. It can be used to infer the mass density parameter and the cosmological constant. As we discuss later (section 4.1) the observation favours a low $\Omega$ and a positive $\lambda$. If we take their formal errors, $\Omega<0.1$ is allowed only at three sigma for a zero $\lambda$ universe (Riess et al. 1998; Perlmutter et al. 1999). A zero $\lambda$ open universe may not be excluded yet if some allowance is taken for systematic effects, but EdS geometry is far away from the observation. The best favoured value is approximately,

$$
\Omega \approx 0.8 \lambda-0.4
$$

\footnotetext{
${ }^{3}$ They suggest $\Omega \simeq 0.04$ on the basis that only early-type galaxy population traces the mass distribution and the luminosity density is multiplied by the fraction of early-type galaxies (20\%). It seems possible that late type galaxies reside in low density regions, causing only a small shear, which is buried in noise, and escaped from the measurement.
} 


\subsubsection{Baryon fractions}

A cluster is a virialised object with the cooling time scale longer than the dynamical time scale, and hence the physics is governed only by gravity (except for cooling flows in high density regions). The gas in clusters is shock heated to the virial temperature $T \simeq 7 \times 10^{7}\left(\sigma / 1000 \mathrm{~km} \mathrm{~s}^{-1}\right)^{2} \mathrm{~K}$, and thus emits $\mathrm{X}$ rays by thermal bremsstrahlung. From the luminosity and temperature of $\mathrm{X}$ rays one can infer the mass of the $\mathrm{X}$ ray emitting gas. It has been known that the gas amounts to a substantial fraction of the dynamical mass, which means that baryons reside more in the gas than in stars by an order of magnitude (Forman \& Jones 1982). The argument was then elaborated by White et al. (1993b) based on ROSAT observations. From 19 clusters White \& Fabian (1995) obtained $M_{\text {gas }} / M_{\text {grav }}=0.056 h^{-2 / 3}$, where $M_{\text {grav }}$ is the dynamical mass. By requiring that the cluster baryon fraction agrees with $\Omega_{B} / \Omega$ in the field, we have $\Omega=0.066 h^{-1 / 2} \eta_{10}=0.39\left(\eta_{10} / 5\right)$, where $\eta_{10}$ is the baryon to photon ratio in units of $10^{-10}$ and the last number assumes $h=0.7$.

An independent estimate is made from the Zeldovich-Sunyaev effect observed in clusters (Myers et al. 1997; Grego et al. 1999): $M_{\text {gas }} / M_{\text {grav }}=$ $0.082 h^{-1}$ is translated to $\Omega=0.044 h^{-1} \eta_{10}=0.31\left(\eta_{10} / 5\right)$.

If we insert a probable value of the baryon to photon ratio from primordial nucleosynthesis calculations, $\eta_{10}=3-5$, we have $\Omega=0.2-0.4$.

\subsubsection{Peculiar velocity - density relation}

This is one of the most traditional methods to estimate the cosmic mass density. The principles are spelled out by Peebles (1980). There are two basic tools depending on the scale. For small scales $(r<1 \mathrm{Mpc})$ the perturbations developed into a non-linear regime, and the statistical equilibrium argument is invoked for ensemble averages that the peculiar acceleration induced by a pair of galaxies is balanced by relative motions (cosmic virial theorem). For a large scale $(r>10 \mathrm{Mpc})$, where perturbations are still in a linear regime, the basic equation is

$$
\nabla \cdot \vec{v}+H_{0} \Omega^{0.6} \delta=0
$$

with $\delta$ the density contrast. The contribution from a cosmological constant is negligible. The problem inherent in all arguments involving velocity is the uncertainty regarding the extent to which galaxies trace the mass distribution (biasing), or how much mass is present far away from galaxies.

Small-scale velocity fields: The status is summarised in Peebles (1999), where he has concluded $\Omega(10 \mathrm{kpc} \lesssim r \lesssim 1 \mathrm{Mpc})=0.15 \pm 0.10$ from the pair wise velocity dispersion (with samples excluding clusters) and the three point correlation function of galaxies via a statistical stability argument. 
Bartlett \& Blanchard (1996) argued that it is possible to reconcile the observed velocity dispersion with $\Omega \sim 1$ if one assumes galactic halo extended beyond $>300 \mathrm{kpc}$. As Peebles (1999) argued, however, the halo is unlikely to be extended that much as indicated by the agreement of MW's mass at $100-200 \mathrm{kpc}$ and the mass estimate for MW+M31 in the Local Group.

Beyond a $10 \mathrm{Mpc}$ scale, linear perturbation theory applies. An integral form of (12) for a spherical symmetric case $\left(v / H_{0} r=\Omega^{0.6}\langle\delta\rangle / 3\right)$ applied to the Virgocentric flow gives $\Omega \simeq 0.2$ for $v \simeq 200-400 \mathrm{~km} \mathrm{~s}^{-1}$ and $\langle\delta\rangle \sim 2$, assuming no biasing (Davis and Peebles 1983). Recently, Tonry et al. (1999) argued that the peculiar velocity ascribed to Virgo cluster is only $140 \mathrm{~km}$ $\mathrm{s}^{-1}$, while the rest of the peculiar velocity flow is attributed to the Hyd-Cen supercluster and the quadrupole field. For this case $\Omega \simeq 0.06$. We may have $\Omega \sim 1$ only when half the mass is well outside the galaxies.

Peebles (1995) argued that the configuration and kinematics of galaxies are grown following the least action principle from the nearly homogeneous primeval mass distribution. Applying this formalism to Local Group galaxies, he inferred $\Omega=0.15 \pm 0.15$. On the other hand, Branchini \& Carlberg (1994) and Dunn \& Laflamme (1995) argued that this conclusion is not tenable if mass is distributed smoothly outside galaxies as in $\Omega=1 \mathrm{CDM}$ models. This seems, however, not very likely unless mass distribution is extended over $10 \mathrm{Mpc}$ scale (Peebles 1999).

Large scale velocity fields: There are a few methods to analyse the largescale velocity fields based on (12). The direct use of (12) is a comparison of the density field derived from redshift surveys with measured peculiar velocities. Alternatively, one may use the density field reconstructed from observed velocity field for comparison with the actual density field, as in the POTENT programme (Dekel et al. 1990). A variant of the first method is to observe the anisotropy in redshift space (redshift distortion) (Kaiser 1987). As linear theory applies, $\Omega$ always appears in the combination $\beta=\Omega^{0.6} / b$ where $b$ is a linear biasing factor of galaxies against the mass distribution and can be inferred through non-linear effects. Much effort has been invested in such analyses (see e.g., Strauss \& Willick 1995; Dekel et al. 1997; Hamilton 1998), but the results are still controversial. The value of $\Omega^{0.6} / b$ derived from many analyses varies from 0.3 to 1.1, though we see a general trend to favour a high value. Notably, the most recent POTENT analysis using the Mark III compilation of velocities (Willick et al. 1997) indicates a high density universe $\Omega=0.5-0.7$, and $\Omega>0.3$ only at a $99 \%$ confidence level (Dekel et al. 1999).

The difficulty is that one needs accurate information for velocity fields, for which an accurate estimate of the distances is crucial. Random errors of the distance indicators introduce large noise in the velocity field. This 
seems particularly serious in the POTENT algorithm, in which the derivative $\nabla \cdot \vec{v} / \Omega^{0.6}$ and its square are numerically computed; this procedure enhances noise, especially for a small $\Omega$. The difficulty of inferring large scale velocity field may also be represented by the 'great attractor problem'. LyndenBell et al. (1988) found a large-scale velocity field towards the Hyd-Cen supercluster, but also argued that this supercluster is also moving towards the same direction attracted by a 'great (giant) attractor'. With Tonry et al.'s (1999) new estimate of the distance using SBF, this velocity field is modest, and Hyd-Cen itself serves as the great attractor that pulls the Virgo cluster, with the conclusion that $\Omega$ is small.

\subsection{MODEL-DEPENDENT DETERMINATIONS}

The following derivations of the mass density parameter are based on the hierarchical clustering model of cosmic structure formation assuming the cold dark matter dominance. The extraction of $\Omega$ is, therefore, indirect, but on the other hand, it is reasonable to appeal to such models since $\Omega$ is the parameter that predominantly controls structure formation. Note that CDM model is the only model known today that successfully predicts widely different observations, yet there are no observations strong enough to refute its validity. We do not discuss results from cosmological models where physical processes other than gravity play a major role.

\subsubsection{Shape parameter of the transfer function}

The initial perturbations of the density fluctuation $P(k)=\left|\delta_{k}\right|^{2} \sim k^{n}$ receive a modification as $P(k)=\left|\delta_{k}\right|^{2} \sim k^{n} T(k)$ as they grow, where $T(k)$ is called the transfer function. Fluctuations of a small scale that enter the horizon in the radiation dominant epoch do not grow for a while, till the universe becomes matter dominated. The transfer function $T(k)$ thus damps for small scales as $\sim k^{-4}$, whereas it stays close to unity for long-wave lengths. The transition region is controlled by a parameter $k \sim 2 \pi / c t_{\text {eq }}$, $c t_{e q}$ being the horizon size at the time of matter-radiation equality, i.e., a characteristic length of $6.5(\Omega h)^{-1} h^{-1} \mathrm{Mpc}$. The parameter $\Gamma=\Omega h$ determines the behaviour of the transfer function and is called the shape parameter. To give a sufficient power to several tens of Mpc, $\Gamma$ must be as small as 0.2 (Efstathiou et al. 1990). This small value $(\Gamma=0.15-0.25)$ is supported by later analyses (e.g., Peacock \& Dodds 1994; Eke et al. 1998).

\subsubsection{Evolution of the rich cluster abundance}

The cluster abundance at $z \approx 0$ requires the rms mass fluctuation $\sigma_{8}=$ $\left.\langle(\delta M / M)\rangle^{1 / 2}\right|_{r=8 h^{-1} \mathrm{Mpc}}$ to satisfy (White et al. 1993a; Eke et al. 1996; Pen 1998; Viana \& Liddle 1999; see also Henry \& Arnaud 1991) 


$$
\sigma_{8} \approx 0.6 \Omega^{-0.5}
$$

The evolution of the cluster abundence is sensitive to $\sigma_{8}$ in early epochs of growth for a given mass; it is $z \gtrsim 0.3$ for rich clusters. The rich cluster abundance at $z \sim 0.3-1$, when compared with that at a low $z$, determines both $\sigma_{8}$ and $\Omega$ (Oukbir \& Blanchard 1992). Carlberg et al. (1997b) derived $\Omega=0.4 \pm 0.2$, and Bahcall \& Fan (1998) obtained $\Omega=0.2_{-0.1}^{+0.3}$ corresponding to a slow growth of the abundance. On the other hand, Blanchard \& Bartlett (1998) obtained $\Omega \simeq 1$ from a more rapid growth. A high value is also claimed by Reichart et al. (1999), while Eke et al. (1998) reported $\Omega=0.43 \pm 0.25$ for an open, and $\Omega=0.36 \pm 0.25$ for a flat universe.

The controversy among authors arises from different estimates of the cluster mass at high $z$. This is a subtle effect, since the mass varies little over the range of relevant redshift, while the cluster number density evolution is sufficiently rapid at fixed mass (Pen 1998). At low $z$ we have an established mass temperature relation, and the cluster mass is securely estimated (Henry \& Arnaud 1991). At high $z$, however, such direct information is not available. Blanchard \& Bartlett and Eke et al. used mass temperature relations as a function of $z$ derived from hydrodynamic simulations. Reichart et al. used an extrapolated mass X-ray luminosity relation. Bahcall and Fan used more direct estimates of the cluster mass at higher $z$ for three clusters. A change of a factor of two in the mass estimate would modify the conclusion.

\subsubsection{Cluster abundance versus the COBE normalisation}

There are a number of ways to infer $\sigma_{8}$ from galaxy clustering and peculiar velocity fields. The problem with the information from galaxy clustering is that it involves an unknown biasing factor, which hinders us from determining an accurate $\sigma_{8}$. The velocity data are susceptible to noise from the distance indicators. Therefore, the cluster abundance discussed above seems to give us a unique method to derive an accurate estimate of $\sigma_{8}$ for a low $z$ universe. Another place we can extract an accurate $\sigma_{8}$ is the fluctuation power imprinted on cosmic microwave background radiation (CBR) anisotropies. Currently only the COBE observation (Bennett et al. 1996) gives sufficiently accurate $\sigma_{8}=\sigma_{8}\left(H_{0}, \Omega, \lambda, \Omega_{B}, \ldots\right)$. Assuming the model transformation function, the matching of $\mathrm{COBE} \sigma_{8}$ with that from the cluster abundance gives a significant constraint on cosmological parameters $\Omega=\Omega\left(H_{0}, \lambda\right)$ (Efstathiou et al. 1992; Eke et al. 1996)Figure 4 shows allowed regions for two cases, open and flat universes, assuming a flat perturbation spectrum $n=1$ and ignoring possible tensor perturbations.

The transfer function is modified if $n \neq 1$. The possible presence of the tensor perturbations in CBR anisotropies causes another uncertainty. The 


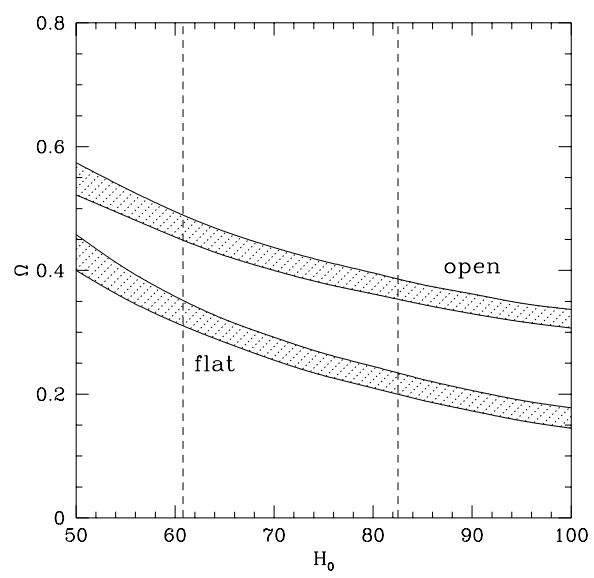

Figure 4. Parameter regions allowed by matching the rms fluctuations from COBE with those from the cluster abundance. A flat spectrum $(n=1)$ is assumed and the tensor perturbations are neglected. The lower band is for a flat universe, and the upper one for a universe with $\Lambda=0$.

COBE data alone say $n$ being between 0.9 and 1.5 (Bennett et al. 1996), but the allowed range is narrowed to $n=0.9-1.2$ if supplemented by smaller angular-scale CBR anisotropy data (Hancock et al. 1998; Lineweaver 1998; Efstathiou et al. 1998; Tegmark 1999). The presence of the tensor mode would make the range of $n$ more uncertain as well as it reduces the value of $\sigma_{8}$. The limit of $n$ when the tensor mode is maximally allowed is about $<1.3^{4}$. Notwithstanding these uncertainties, $\Omega>0.5$ is difficult to reconcile with the matching condition. On the other hand, a too small $\Omega(\lesssim 0.15)$ is not consistent with the cluster abundance.

\subsubsection{Power spectrum in nonlinear galaxy clustering}

Peacock (1997) argued that the power spectrum in a small scale region $\left(k^{-1}<3 h^{-1} \mathrm{Mpc}\right)$, where nonlinear effect is dominant, shows more power than is expected in $\Omega=1$ cosmological models. He showed that the excess power is understood if the mass density is $\Omega \approx 0.3$.

\subsubsection{CBR anisotropy harmonics}

The $\ell$ distribution of the CBR harmonics $C_{\ell}$ depends on many cosmological parameters. Precise measurements of the harmonics will allow an accurate determination of the cosmological parameters up to geometrical degeneracy (Zaldarriaga et al. 1997; Efstathiou \& Bond 1999; Eisenstein et al. 1999). At

\footnotetext{
${ }^{4}$ In Tegmark's analysis $n<1.5$ is quoted as an upper bound, but this is obtained by making $\Omega_{B}$ (and $H_{0}$ ) a free parameter. If one would fix the baryon abundance, the allowed range is narrower, $n \lesssim 1.3$.
} 
present the data do not give any constraint on $\Omega$, but on some combination of $\Omega$ and $\lambda$; so we defer the discussion to the next section.

\section{Cosmological constant}

Currently three tests yield useful results on the problem as to the existence of the cosmological constant: (i) the Hubble diagram for distant type Ia supernovae; (ii) gravitational lensing frequencies for quasars; (iii) position of the acoustic peak in the harmonics of CBR anisotropies.

\subsection{TYPE IA SUPERNOVA HUBBLE DIAGRAM}

The luminosity distance receives a cosmology dependent correction as $z$ increases; in a way $\Omega$ pulls down $d_{L}$ and $\lambda$ pushes it up. (In the first order of $z$ the correction enters in the combination of $q_{0}=\Omega / 2-\lambda$, so this is often referred to as a $q_{0}$ test.) The discovery of two groups that distant supernovae are fainter than are expected from the local sample, even fainter than are expected for $q_{0}=0$, points to the presence of $\lambda>0$ (Riess et al. 1998; Schmidt et al. 1998; Perlmutter et al. 1999).

The general difficulty with such a Hubble diagram analysis is that one has to differentiate among a few interesting cosmologies with small differences of brightness. For instance, at $z=0.4$ where many supernovae are observed, the difference is $\Delta m=0.12$ mag between $(\Omega, \lambda)=(0.3,0.7)$ and $(0,0)$, and $\Delta m=0.22$ from $(0,0)$ to $(1.0,0)$. Therefore, an accuracy of $(\lesssim 5 \%)$ must be attained including systematics to conclude the presence of $\Lambda$. On the other hand, there are a number of potential sources of errors:

(i) K corrections evaluated by integrating spectrophotometric data that are dominated by many strong features;

(ii) relative fluxes at the zero point (zero mag) across the colour bands;

(iii) dust obscuration in a host galaxy;

(iv) subtraction of light from host galaxies;

(v) identification of the maximum brightness epoch, and estimates of the maximum brightness including a $\Delta m_{15}$ correction;

(vi) selection effects (for high $z \mathrm{SNe}$ );

(vii) evolution effects.

Except for (vii), for which we cannot guess much ${ }^{5}$, the most important seems to be combined effects of (i), (ii) and (iii). It is not easy a task to reproduce a broad band flux by integrating over spectrophotometric data convoluted with filter response functions, especially when spectrum

\footnotetext{
${ }^{5}$ Riess et al. (1999) showed that the rise time is different between low $z$ and high $z$ samples, indicating some evolution of the SNIa population. The effect on the cosmological parameter is not clear.
} 
TABLE 8. Estimates of maximum brightness on SNe: 1997 vs. 1999 from Perlmutter et al. (1997; 1999).

\begin{tabular}{llll}
\hline SN & 1997 value & 1999 value & difference \\
\hline SN1992bi & $(23.26 \pm 0.24)$ & $23.11 \pm 0.46$ & $(0.15)$ \\
SN1994H & $22.08 \pm 0.11$ & $21.72 \pm 0.22$ & 0.36 \\
SN1994al & $22.79 \pm 0.27$ & $22.55 \pm 0.25$ & 0.24 \\
SN1994F & $(21.80 \pm 0.69)$ & $22.26 \pm 0.33$ & $(-0.58)$ \\
SN1994am & $22.02 \pm 0.14$ & $22.26 \pm 0.20$ & -0.24 \\
SN1994G & $22.36 \pm 0.35$ & $22.13 \pm 0.49$ & 0.23 \\
SN1994an & $22.01 \pm 0.33$ & $22.58 \pm 0.37$ & -0.57 \\
\hline
\end{tabular}

Note: The numbers in the parentheses are not used in the final result of the 1997 paper.

contains strong features. (Even for the spectrophotometric standard stars, the synthetic magnitude contains an error of 0.02-0.05 mag, especially when the colour band involves the Balmer or Paschen regions.) Whereas Perlmutter et al. assigns 0.02 mag to the error of (i) [and (ii)], a comparison of the two values of estimated maximum brightness in their 1997 paper (Perlmutter et al. 1997, where they claimed evidence for a high $\Omega$ universe) and the 1999 paper (TABLE 8) shows a general difficulty in the evaluation of the $K$ correction (the difference dominantly comes from different $K$ corrections). Schmidt et al. claim that their K correction errors are $0.03 \%$ mag. Dust obscuration (iii) is also an important source of errors, since the error of (i)+(ii) propagates to $E(B-V)$ and is then amplified with the $R$ factor. So a $0.02 \mathrm{mag}$ error in colour results in a $0.06 \mathrm{mag}$ error in $A_{V}$.

We note that each SN datum contains $\pm 0.2 \mathrm{mag}$ (20\%) error. The issue is whether this error is almost purely of random nature and systemtics are controlled to a level of $\lesssim 0.05$.

\subsection{GRAVITATIONAL LENSING FREQUENCIES FOR QUASARS}

The gravitational lensing optical depth is given by

$$
d \tau=\mu F H_{0}^{3}\left(1+z_{L}\right)^{3}\left[\frac{D_{O L} D_{L S}}{D_{O S}}\right]^{2} \frac{d t}{d z} d z
$$

where $F=\left\langle 16 \pi^{3} n_{g} \sigma_{g}^{4} H_{0}^{-3}\right\rangle$, and $\mu$ is a magnification factor. The cosmological factor in (14) is very sensitive to the cosmological constant, when it dominates (Fukugita \& Turner 1991). $F$ is the astrophysical factor that depends on the galaxy number density $n_{g}$ and the mass distribution of galaxies, which is usually assumed to be a singular isothermal sphere with velocity dispersion $\sigma_{g}$. Figure 5 shows a typical calculation for the expected 


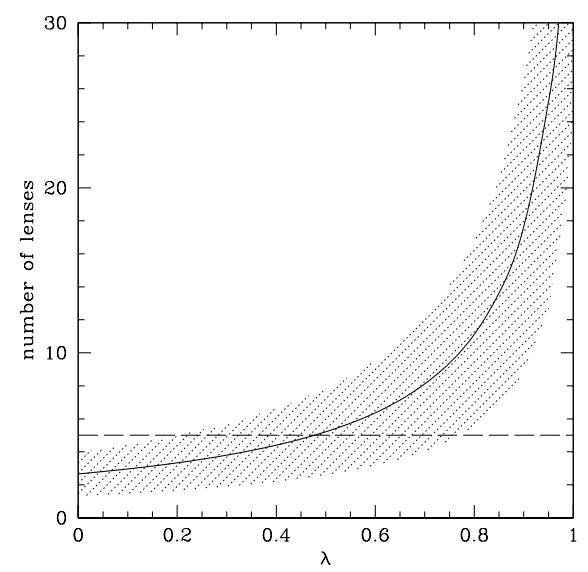

Figure 5. Gravitational lensing frequencies as a function of $\Lambda$ in a flat universe. The expecetd number is given for 504 quasars of the HST Snapshot Survey sample. The shade means the region within a $\pm 50 \%$ uncertainty. The observed number is 5 (dashed line).

number of strong lenses for 504 quasars of the HST Snapshot Survey (Maoz et al. 1993) sample: the observed number is 5 (4 if $0957+561$ is excluded). The curve shows a high sensitivity to $\lambda$ for $\lambda>0.7$, but in contrast a nearly flat dependence for a lower $\lambda$. It is likely that $\lambda>0.8$ is excluded. On the other hand, a more stringent limit is liable to be elusive. Fifty percent uncertainty in the $F$ factor, say, would change largely a limit on, or a likely value of, $\lambda$.

In order to acquire information for a smaller $\lambda$, an accurate estimate is essential for the $F$ factor, which receives the following uncertainties in: (1) the luminosity density and the fraction of early-type galaxies (the lensing power of $\mathrm{E}$ and $\mathrm{S} 0$ galaxies is much higher than that of spirals, and $F$ is roughly proportional to the luminosity density of early-type galaxies); (2) $\sigma_{g}$-luminosity relation (Faber-Jackson relation); (3) the relation between $\sigma$ (dark matter) and $\sigma$ (star); (4) the model profile of dark haloes, specifically the validity of the singular isothermal sphere approximation (note that dark matter distributions seem more complicated in elliptical galaxies than in spiral galaxies, see Fukugita \& Peebles 1999); (5) the core radius which leads to a substantial reduction in $d \tau$; $(6)$ selection effects of the observations; (7) dust obscuration; (8) evolution of early-type galaxies.

There are continuous efforts for nearly a decade that have brought substantial improvement in reducing these uncertainties (Maoz \& Rix 1993; Kochanek 1996; Falco et al. 1998). Nevertheless, the issue (1) still remains as a cause of a large uncertainty. While the total luminosity density is known to an uncertainty of $20 \%$ or so, the fraction of early type galaxies is more uncertain. It varies from 0.20 to 0.41 depending on the literature. 
Including other items, it is likely that an estimate of $F$ has a $50 \%$ uncertainty. For the curve in Figure 5 a change of $F$ by $\pm 50 \%$ brings the most likely value of $\lambda$ to 0.75 or 0.2 .

Kochanek and collaborators have made detailed considerations on the above uncertainties, and carried out elaborate statistical analyses. In their latest publication they concluded $\lambda<0.62$ at $95 \%$ confidence level from an optical sample (Kochanek 1996). They took the fraction of early-type galaxies to be 0.44 and assigned a rather small $1 \sigma$ error. (The predicted frequency comes close to the upper envelope of Fig. 5, and the observed number of lenses in the HST sample is taken to be 4). If one would adopt a smaller early-type fraction, the limit is immediately loosened by a substantial amount. Since the uncertainty is dominated by systematics rather than statistical, it seems dangerous to give significance to statistics. Statistical significance depends on artificial elements as to what are assumed in the input. A similar comment also applies to the recent work claiming for a positive $\lambda$ (Chiba \& Yoshii 1997; Cheng \& Krauss 1998). I would conclude a conservative limit being $\lambda<0.8$.

\subsection{HARMONICS OF CBR ANISOTROPIES}

This is a topic discussed repeatedly in this Summer Institute, so I will only briefly mention it for completeness. The positions of the acoustic peaks are particularly sensitive to $\Omega$ and $\lambda$, and even low accuracy data available at present lead to a meaningful constraint on a combination of $\Omega$ and $\lambda$.

The first acoustic peak appears at $\ell=\pi$ (the distance to the lastscattering surface)/(the sound horizon) (Hu \& Sugiyama 1995). Its position $\ell_{1}$ is approximated as

$$
\ell_{1} \simeq 220\left(\frac{1-\lambda}{\Omega}\right)^{1 / 2}
$$

for the parameter range that concerns us. This means that the position of the acoustic peak is about $\ell \simeq 220$ if $\Omega+\lambda=1$, but it shifts to a high $\ell$ as $\Omega^{-1 / 2}$ if $\lambda=0$. On the other hand, there is little power to determine $\Omega$ separately from $\lambda$, unless full information of $C_{\ell}$ is used. The harmonics $C_{\ell}$ measured at small angles revealed the acoustic peak (Scott et al. 1996), and its position favours a universe not far from flat (Hancock et al. 1998). More exhaustive analyses of Lineweaver (1998) and Efstathiou et al. (1999) show a limit $\Omega+\lambda / 2>0.52(1 \sigma)$. (The contours of the confidence level fall approximately on the curve given by (15) with $\ell_{1}=$ constant.) This means that a zero $\Lambda$ universe is already marginal, when combined with $\Omega$ from other arguments. If a flat universe is chosen from CBR, a non-zero $\Lambda$ will be compelling. 
TABLE 9. Summary of $\Omega$ and $\lambda$.

\begin{tabular}{|c|c|c|c|}
\hline method & $\Omega_{0}$ & $\Lambda ?$ & model used? \\
\hline$H_{0}$ vs $t_{0}$ & $<0.7$ & & \\
\hline luminosity density $+\mathrm{M} / \mathrm{L}$ & $0.1-0.4$ & & \\
\hline cluster baryon fraction & $0.15-0.35$ & & \\
\hline SNeIa Hubble diagram & $\leq 0.3$ & $\lambda \approx 0.7$ & \\
\hline small-scale velocity field (summary) & $0.2 \pm 0.15$ & & \\
\hline (pairwise velocity) & $0.15 \pm 0.1$ & & \\
\hline (Local Group kinematics) & $0.15 \pm 0.15$ & & \\
\hline (Virgocentric flow) & $0.2 \pm 0.2$ & & \\
\hline large-scale vel field & $0.2-1$ & & \\
\hline cluster evolution (low $\Omega$ sol'n) & $0.2_{-0.1}^{+0.3}$ & & yes \\
\hline$($ high $\Omega$ sol'n $)$ & $\sim 1$ & & yes \\
\hline \multirow[t]{2}{*}{ COBE-cluster matching } & $0.35-0.45$ (if $\lambda=0)$ & & yes \\
\hline & $0.20-0.40($ if $\lambda \neq 0)$ & & yes \\
\hline shape parameter $\Gamma$ & $0.2-0.4$ & & yes \\
\hline \multirow[t]{2}{*}{ CBR acoustic peak } & free (if flat) & $\gtrsim 1-2 \Omega$ & yes \\
\hline & $>0.5$ (if open) & & yes \\
\hline gravitational lensing & & $\lambda<0.8$ & \\
\hline \multirow[t]{3}{*}{ summary } & $0.15-0.45$ (if open) & & \\
\hline & $0.2-0.4$ (if flat) & & \\
\hline & & $0.6-0.7(?)$ & \\
\hline
\end{tabular}

\section{Conclusions}

The status of $\Omega$ and $\lambda$ is summarised in TABLE 9 . We have a reasonable convergence of the $\Omega$ parameter towards a low value $\Omega=0.15-0.4$. The convergence of $\Omega$ is significantly better with the presence of the cosmological constant that makes the universe flat. Particularly encouraging is that the $\Omega$ parameters derived with the aid of structure formation models agree with each other. This is taken to be an important test for the cosmological model, just as in particle physics when many different phenomena are reduced to a few convergent parameters to test the model. There are yet a still highly discrepant results on $\Omega$, but it is not too difficult to speculate their origins. On the other hand, the current 'low $\Omega$ ' means the values that vary almost by a factor of three and effort is needed to make these converge.

The cosmological constant has been an anathema over many years because of our ignorance of any mechanism that could give rise to a very small vacuum energy of $(3 \mathrm{meV})^{4}$, and neither can we understand a zero cosmo- 

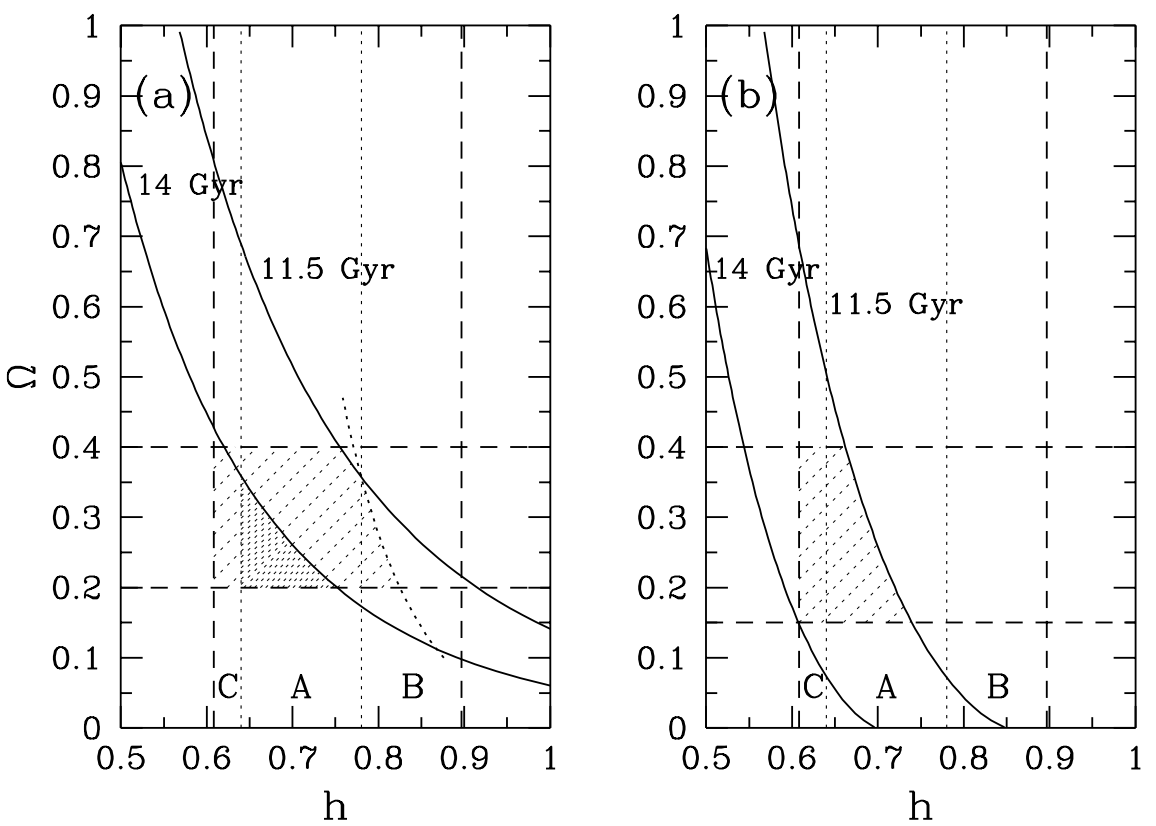

Figure 6. Consistent parameter ranges in the $H_{0}-\Omega$ space for (a) a flat universe and (b) an open universe. $A$ is the range of the Hubble constant when $(m-M)_{\mathrm{LMC}}=18.5 . B$ or $C$ is allowed only when the LMC distance is shorter by $0.3 \mathrm{mag}$, or longer by $0.1 \mathrm{mag}$. Note in panel (a) that most of the range of $B$ is forbidden by the compatibility of age and $H_{0}$ that are simultaneously driven by the RR Lyr calibration (section 2.7). Also note that the age range between $\approx 11.5 \mathrm{Gyr}$ and $\approx 14 \mathrm{Gyr}$ is possible only with the interpretation that globular cluster formation is coevel (section 2.4). The most naturally-looking parameter region is given a thick shade.

logical constant. In mid-nineties the atmosphere was changing in favour for a non-zero $\Lambda$. The prime motivation was the Hubble constant-age problem, but the introduction of a non-zero $\Lambda$ was helpful in many respects. One theoretical motivation was to satisfy flatness which is expected in inflationary scenarios (Peebles 1984). Ostriker \& Steinhardt (1995) proclaimed a 'cosmic concordance' with a flat universe mildly dominated by $\Lambda$. By 1997, only one observation contradicted with the presence of a moderate value of $\Lambda$; this was the SNeIa Hubble diagram presented by the Supernova Cosmology Project (Perlmutter et al. 1997); see Fukugita 1997. In the next two years the situation changed. Two groups analysing SNeIa Hubble diagram, including the Supernova Cosmology Project, now claim a low $\Omega$ and a positive $\Lambda$. On the other hand, the Hubble constant-age problem became less severe due to our cognition of larger uncertainties, especially in the age estimate. The indications from SNeIa Hubble diagram are very interest- 
ing and important, but the conclusions are susceptible to small systematic effects. They should be taken with caution. We should perhaps wait for small-scale CBR anisotropy observations to confirm a nearly flat universe before concluding the presence of $\Lambda$.

In these lectures we have not considered classical tests, number counts, angular-size redshift relations, and magnitude-redshift relations of galaxies (Sandage $1961 ; 1988$ ), in those testing for $\Omega$ and $\Lambda$. Unlike clusters or large scale structure, where no physics other than gravity plays a role, the evolution of galaxies is compounded by rich physics. Unless we understand their astrophysics, these objects cannot be used as testing candles. It has been known that galaxy number counts is understood more naturally with a low matter density universe under the assumption that the number of galaxies are conserved, but it is possible to predict the correct counts with an $\Omega=1$ model where galaxies form through hierarchical merging, by tuning parameters that control physics (Cole et al. 1994; Kauffmann et al. 1994). It is important to work out whether the model works for any cosmological parameters or it works only for a restricted parameter range. This does not help much to extract the cosmological parameters, but it can falsify the model itself.

We have seen impressive progress in the determination of the Hubble constant. The old discrepancy is basically solved. On the other hand, a new uncertainty emerged in more local distance scales. The most pressing issue is to settle the value of the distance to LMC. There are also a few issues to be worked out should one try to determine $H_{0}$ to an accuracy of a $10 \%$ error or less. They include understanding of metallicity effects and interstellar extinction. The future effort will give more weight to geometric or semi-geometric methods. From the view point of observations the work will go to infra-red colour bands to minimise these problems.

In conclusion, I present in Figure 6 allowed ranges of $H_{0}$ and $\Omega$ (and $\lambda$ ) for the case of (a) flat and (b) open universes. With the flat case we cut the lower limit of $\Omega$ at 0.2 due to a strong constraint from lensing. An ample amount of parameter space is allowed for a flat universe. A high value of $H_{0}>82$, which would be driven only by a short LMC distance, is excluded by consistency with the age of globular clusters as noted earlier. Therefore, we are led to the range $H_{0} \simeq 60-82$ from the consistency conditions. For an open universe the coeval-formation interpretation is compelling for globular clusters, or else no region is allowed. The allowed $H_{0}$ is limited to $60-70$. No solution is available if LMC takes a short distance.

I would like to thank Rob Crittenden for his careful reading and many useful suggestions on this manuscript. This work is supported in part by Grant in Aid of the Ministry of Education in Tokyo and Raymond and 
Beverly Sackler Fellowship in Princeton.

\section{References}

Aaronson, M. et al. 1986, ApJ, 302, 536

van Altena,, B. et al. 1997, Baltic Astron., 6(1), 27

Andersen, J. 1991, A\& AR, 3, 91

Arnett, W. D., Branch, D. \& Wheeler, J. C. 1985, Nature, 314, 337

Bahcall, N. A. \& Fan, X. 1998, ApJ, 504, 1

Bahcall, N. A., Lubin, L. M. \& Dorman, V. 1995, ApJ, 447, L81

Bartlett, J. G. \& Blanchard, A. 1996, A\& A, 307, 1

Beaulieu et al. 1997, A\& A, 318, L47

Bell, S. A., Hill, G., Hilditch, R. W., Clausen, J. V. \& Reynolds, A. P. 1993, MNRAS, 265, 1047

Bennett, C. L. et al. 1996, ApJ. 464, L1

van den Bergh, S. 1989, A\& AR, 1, 111

van den Bergh, S. 1994, PASP, 106, 1113

van den Bergh, S. 1995, ApJ, 446, 39

Bernstein, G. \& Fischer, P. 1999, astro-ph/9903274

Biggs, A. D. et al. 1999, MNRAS, 304, 349

Birkinshaw, M., Hughes, J. P. \& Arnaud, K. A. 1991, ApJ, 379, 466

Blakeslee, J. P., Ajhar, E. A. \& Tonry, J. L. 1998, in Post Hipparcos Cosmic Candles, eds. A. Heck \& F. Caputo (Kluwer, Boston), p.181

Blanchard, A. \& Bartlett, J. G. 1998, A\& A, 332, L49

Bolte, M. \& Hogan, C. J. 1995, Nature, 376, 399

Branch, D. 1992, ApJ, 392, 35

Branch, D. \& Miller, D. L. 1993, ApJ, 405, L5

Branchini, E. \& Carlberg, R. G. 1994, ApJ, 434, 37

Buonanno, R., Corsi, C. E. \& Fusi Pecci, F. 1989, A\& A, 216, 80

Caldwell, J. A. R. 1983, Observatory, 103, 244

Carlberg, R. G., Yee, H. K. C. \& Ellingson, E. 1997a, ApJ, 478, 462

Carlberg, R. G., Morris, S. L., Yee, H. K. C. \& Ellingson, E. 1997b, ApJ, 479, L19

Carlberg, R. G. et al. 1996, ApJ, 462, 32

Chaboyer, B., Demarque, P. \& Sarajedini, A. 1996, ApJ, 459, 558

Chaboyer, B., Demarque, P. Kernan, P. J. \& Krauss, L. M. 1998, ApJ, 494, 96

Cheng, Y.-C. N. \& Krauss, L. M. 1998, astro-ph/9810393

Chiba, M. \& Yoshii, Y 1997, ApJ, 490, L73

Chiosi, C., Wood, P. R. \& Capitanio, N. 1993, ApJS, 86, 541

Ciardullo, R., Jacoby, J. H. \& Tonry, J. L. 1993, ApJ, 419, 479

Cole, A. A. 1998, ApJ 500, L137

Cole, S. et al. 1994, MNRAS, 271, 781

Conti, P. S., Garmany, C. D. \& Massey, P. 1986, AJ, 92, 48

Courbin, F. et al. 1997, A\& A, 324, L1

Davis, M. \& Peebles, P. J. E. 1983, ARA\& A, 21, 109

Dekel, A., Bertschinger, E \& Faber, S. M. 1990, ApJ, 364, 349

Dekel, A., Burstein, D. \& White, S. D. M. 1997, in Critical Dialogues in Cosmology, ed. N. Turok (World Scientific, Singapore), p. 175

Dekel, A. et al. 1999, ApJ, 522, 1

Dunn, A. M. \& Laflamme, R. 1995, ApJ, 443, L1

Efstathiou, G. \& Bond, J. R. 1999, MNRAS, 304, 75

Efstathiou, G., Bond, J. R. \& White, S. D. M. 1992, MNRAS, 258, 1p

Efstathiou, G., Sutherland, W. J. \& Maddox, S. J. 1990, Nature, 348, 705

Efstathiou, G., Bridle, S. L., Lasenby, A. N., Hobson, M. P. \& Ellis, R. S. 1999, MNRAS, 303,47 
Eisenstein, D. J., Hu, W. \& Tegmark, M. 1999, ApJ. 518, 2

Eke, V. R., Cole, S., \& Frenk, C. S. 1996, MNRAS, 282, 263

Eke, V. R., Cole, S., Frenk, C. S. \& Henry, J. P. 1998, MNRAS, 298, 1145

ESA 1997, The Hipparcos and Tycho Catalogues SP-1200 (ESA, Noordwijk)

Faber, S. M. \& Gallagher, J. S 1979, ARA\& A, 17, 135

Falco, E. E., Gorenstein, M. V.\& Shapiro, I. I. 1991, ApJ, 372, 364

Falco, E. E., Kochanek, C. S. \& Muñoz, J. A. 1998, ApJ, 494, 47

Feast, M. W. \& Catchpole, R. M. 1997 MNRAS, 286, L1

Feast, M. W. \& Walker, A. R. 1987, ARA\& A, 25, 345

Federspiel, M., Tammann, G. A. \& Sandage, A, 1998, ApJ 495, 115

Fernley, J. et al. 1998, A\&A, 330, 515

Ferrarese, L et al. 1999a, astro-ph/9908192

Ferrarese, L et al. 1999b, astro-ph/9910501

Forman, W. \& Jones, C. 1982, ARA\& A, 20, 547

Freedman, W. L. \& Madore, B. F. 1990, ApJ, 365, 186

Freedman, W. L., Madore, B. F. \& Kennicutt, R. C. 1997, in The Extragalactic distance scale, eds. M. Livio, M. Donahue, \& N. Panagia (Cambridge University Press, Cambridge), p.171

Freedman, W. L. et al. 1994, Nature, 371, 757

Fry, A. M. \& Carney, B. W. 1997, AJ, 113, 1073

Fukugita, M. 1997, in Critical Dialogues in Cosmology, ed. N. Turok (World Scientific, Singapore, 1997), p.204

Fukugita, M. \& Peebles, P. J. E. 1999, ApJ. 524, L31

Fukugita, M. \& Turner, E. L. 1991, MNRAS, 253, 99

Fukugita, M., Hogan, C. J. \& Peebles, P. J. E. 1993, Nature, 366, 309

Fukugita, M., Hogan, C. J. \& Peebles, P. J. E. 1998, ApJ, 503, 518

Gibson, B. K. et al. 1999, astro-ph/9908149, ApJ, in press

Gieren, W. P., Fouqué, P. \& Gómez, M. 1998, ApJ, 496, 17

Giovanelli, R. et al. 1997, AJ, 113, 22

Gould, A 1994, ApJ, 426, 542

Gould, A \& Popowski, P. 1998, ApJ, 508, 844

Gould, A \& Uza, O. 1998, ApJ, 494, 118

Gratton, R. G. et. al. 1997 ApJ, 491, 749

Grego, L. et al. 1999, presented at the AAS meeting (194.5807G)

Grogin, N. A. \& Narayan, R. 1996, ApJ, 464, 92

Guinan, E. F. et al. 1998, ApJ, 509, L21

Hamilton, A. J. S. 1998, in The Evolving Universe, ed. D. Hamilton, (Kluwer, Dordrecht, 1998), p. 185

Hamuy, M. et al. 1996a, ApJ, 112, 2391

Hamuy, M. et al. 1996b, ApJ, 112, 2398

Hancock, S., Rocha, G., Lasenby, A. N. \& Gutiérrez, C. M. 1998, MNRAS, 294, L1

Henry, J. P. \& Arnaud, K. A. 1991, ApJ, 372, 410

Hanson, R. B. 1980, in Star Clusters, IAU Symposium 85, ed. J. E. Hesser (Reidel, Dordrecht), p. 71

Herrnstein, J. R. et al. 1999, Nature, 400, 539

Hu, W. \& Sugiyama, N. 1995, Phys. Rev., D51, 2599

Hubble, E. P. 1925, Observatory, 48, 139

Iben, I. \& Tuggle, R. S. 1975, ApJ, 197, 39

Impey, C. D. et al. 1998, ApJ, 509, 551

Jacoby, J. H., Ciardullo, R. \& Ford, H. C. 1990a, ApJ, 356, 332

Jacoby, J. H., Walker, A. R. \& Ciardullo, R. 1990b, ApJ, 365, 471

Jacoby, J. H., Ciardullo, R. \& Harris, W. E. 1996, ApJ, 462,1

Jacoby, J. H. et al. 1992, PASP, 104, 599

Jensen, J. B., J. L.Tonry \& Luppino, G. A. 1999, ApJ, 510, 71

Jha, S. et al. 1999, astro-ph/9906220, ApJ in press 
Kaiser, N. 1987, MNRAS 227, 1

Kaiser, N. et al. 1998, astro-ph/9809268

Kauffmann, G., Guiderdoni, B. \& White, S. D. M. 1994, MNRAS, 267, 981

Keeton, C. R. \& Kochanek 1997, ApJ, 487, 42

Kelson, D. D. et al. 1999, astro-ph/9909222, ApJ in press

Kennicutt, R. C. et al. 1998, ApJ, 498, 181

Kochanek, C. S. 1996a, ApJ, 457, 228

Kochanek, C. S. 1996, ApJ, 466, 638

Kochanek, C. S. 1997, ApJ, 491, 13

Koopmans, L. V. E. \& Fassnacht, C. D. 1999, astro-ph/9907258, ApJ, in press

Kovács, J \& Jurcsik, J. 1996, ApJ, 466, L17

Kraan-Korteweg, R. C., Cameron, L. M. \& Tammann, G. A. 1988, ApJ, 331, 620

Kundić, T., Cohen, J. G., Blandford, R. D. \& Lubin, L. M. 1997a, AJ, 114, 507

Kundić, T. et al. 1997b, ApJ, 482, 75

Laney, C. D. \& Stobie, R. S. 1994, MNRAS, 266, 441

Little, B. \& Tremaine, S. 1987, ApJ, 320, 493

van Leeuwen, F. 1983, PhD thesis (Leiden University)

van Leeuwen, F. 1999, A\& A, 341, L71

van Leeuwen, F. \& Hansen-Ruiz, C. S. 1997, in Hipparcos Venice '97, ed. B. Battrick (ESA, Noordwijk, 1997), p. 689

van Leeuwen, F., Feast, M. W., Whitelock, P. A. \& Yudin, B. 1997, MNRAS, 287, 955

Leibundgut, B. \& Pinto, P. A. 1992, ApJ, 401, 49

Lineweaver, C. H. 1998, ApJ, 505, L69

Lovell, J. E. J. et al. 1998, ApJ, 508, L51

Lundqvist, P. \& Sonneborn, G. 1997, in SN1987A, Ten Years After, ed. M. Phillips \& N. Santzeff (ASP, San Francisco, 1997) to be published

Luri, X., Gómez, A. E., Torra, J., Figueras, F. \& Mennessier, M. O. 1998, A\& A, 335, L81

Lynden-Bell, D. et al. 1988, ApJ, 326, 19

Lyngå, G. 1987, Catalogue of Open Cluster Data, 5th edition (Lund University)

Madore, B. F. \& Freedman, W. L. 1991, PASP, 103, 933

Madore, B. F. \& Freedman, W. L. 1998, ApJ, 492, 110

Maoz, D. \& Rix, H.-W. 1993, ApJ, 416, 425

Maoz, D. et al. 1993, ApJ, 402, 69

Maoz, E. et al. 1999, Nature, 401, 351

Mateo, M. \& Hodge, P. 1986, ApJS, 60, 893

Mermilliod, J.-C., Turon, C., Robichon, N., Arenou, F. \& Lebreton, Y. 1997, in Hipparcos Venice '97, ed. B. Battrick (ESA, Noordwijk, 1997), p. 643

Miyoshi, M. et al. 1995, Nature, 373, 127

Mochejska, B. J., Macri, L. M., Sasselov, D. D. \& Stanek, K. Z. 1999, astro-ph/9908293

Myers, S. T., Baker, J. E., Readhead, A. C. S., Leitch, E. M. \& Herbig, T. 1997, ApJ, 485,1

Mould, J. R. et al. 1999, astro-ph/9909260, ApJ, in press

Narayanan, V. K. \& Gould, A. 1999, ApJ, 523, 328

Ostriker, J. P. \& Steinhardt, P. J. 1995, Nature, 377, 600

Oukbir, J. \& Blanchard, A. 1992, A\& A, 262, L21

Paczyński, B. 1997, in The Extragalactic distance scale, eds. M. Livio, M. Donahue, \& N. Panagia (Cambridge University Press, Cambridge), p.273

Paczyński, B. \& Stanek, K. Z. 1998, ApJ, 494, L219

Panagia, N., Gilmozzi, R., Macchetto, F., Adore, H.-M. \& Kirshner, R. P. 1991, ApJ, 380, L23

Panagia, N. , Gilmozzi, R. \& Kirshner, R. 1997, in SN1987A, Ten Years After, ed. M. Phillips \& N. Santzeff (ASP, San Francisco, 1997) to be published

Paturel, G. et al. 1997, in Hipparcos Venice '97, ed. B. Battrick (ESA, Noordwijk, 1997), p. 629. 
Peacock, J. A. 1997, MNRAS, 284, 885

Peacock, J. A. \& Dodds, S. J. 1994, MNRAS, 267, 1020

Peebles, P. J. E. 1980, The Large-Scale Structure of the Universe (Princeton University

Press, Princeton)

Peebles, P. J. E. 1984, ApJ, 284, 439

Peebles, P. J. E. 1995, ApJ, 449, 52

Peebles, P. J. E. 1999, in Formation of Structure in the Universe, eds Dekel, A. \& Ostriker, J. P. (Cambridge University Press, Cambridge), p. 435

Pen, U.-L. 1998, ApJ, 498, 60

Peng et al. 1998, cited in Bridges, T. astro-ph/9811136

Perlmutter, S. et al. 1997, ApJ, 483, 565

Perlmutter, S. et al. 1999, ApJ, 517, 565

Perryman, M. A. C. et. al. 1998, A\& A, 331, 81

Phillips, M. M. 1993, ApJ, 413, L105

Phillips, M. M. et al. 1999, astro-ph/9907052

Pierce, M. J. \& Tully, R. B. 1988, ApJ, 330, 579

Pierce, M. J. et al. 1994, Nature, 371, 385

Pinsonneault, M. H., Stauffer, J., Soderblom, D. R., King, J. R. \& Hanson, R. B. 1998, ApJ, 504, 170

Pskovskiǐ, Yu. P. 1984, Astron. Zh., 61, 1125 (Sov. Astron. 28, 658)

Refsdal, S. 1964, MNRAS, 128, 307

Reichart, D. E. et al. 1999, ApJ, 518, 521

Reid, I. N. 1997, AJ, 114, 161

Renzini, A. 1991, in Observational Tests of Cosmological Inflation, ed. T. Shanks et al. (Kluwer, Dordrecht), p. 131

Riess, A. G., Press, W. H. \& Kirshner, R. P. 1995, ApJ, 438, L17

Riess, A. G., Filippenko, A. V., Li, W. \& Schmidt, B. P. 1999, astro-ph9907038, ApJ, in press

Riess, A. G. et al. 1998, AJ, 116, 1009

Saha, A. et al. 1999, 522, 802

Sakai, S. et al. 1999, astro-ph/9909269

Sandage, A. 1961, ApJ, 133, 355

Sandage, A. 1988, ARA\& A, 26, 561

Sandage, A. 1993a, ApJ, 402, 3

Sandage, A. 1993b, AJ, 106, 703

Sandage, A., Bell, R. A. \& Tripicco, M. J. 1999, ApJ, 522, 250

Sandage, A. \& Tammann, G. A. 1968, ApJ, 151, 531

Sandage, A. \& Tammann, G. A. 1982, ApJ, 256, 339

Sandage, A. \& Tammann, G. A. 1997, in Critical Dialogues in Cosmology, ed. N. Turok

(World Scientific, Singapore, 1997), p. 130

Sandage, A., Saha, A. Tammann, G. A., Panagia, N. \& Macchetto, D. 1992, ApJ, 401, L7

Schechter, P. L. et al. 1997, ApJ, 475, L85

Schmidt, B. P., Kirshner, R. P. \& Eastman, R. G. 1992, ApJ, 395, 366

Schmidt, B. P. et al. 1994, ApJ, 432, 42

Schmidt, B. P. et al. 1998, ApJ, 507, 46

Schommer, R. A., Olszewski, E. W. \& Aaronson, M. 1984, ApJ, 285, L53

Schutz, B. F. 1986, Nature, 323, 310

Scott, P. F. et al. 1996, ApJ, 461, L1

Sekiguchi, M. \& Fukugita, M. 1998, Observatory, 118, 73

Small, T. A., Ma, C.-P., Sargent, W. L. W. \& Hamilton, D 1998, ApJ, 492, 45

Sonneborn, G. et al. 1997, ApJ, 477, 848

Squires, G., Kaiser, N., Fahlman, G., Babul, A. \& Woods, D. 1996, ApJ 469, 73

Stanek, K. Z. \& Garnavich, P. M. 1998, ApJ, 503, L131

Stanek, K. Z., Zaritsky, D. \& Harris, J. 1998, ApJ, 500, L141 
Stothers, R. B. 1988, ApJ, 329, 712

Strauss, M. A. \& Willick, J. A. 1995, Phys. Rep., 261, 271

Suntzeff, N. B. et al. 1999, ApJ, 117, 1175

Szabados, L. in Hipparcos Venice '97, ed. B. Battrick (ESA, Noordwijk, 1997), p. 657

Tammann, G. A. 1999, in Cosmological Parameters and the Evolution of the Universe

(IAU Symposium 183), ed. K. Sato (Kluwer, Dordrecht), p. 31

Tammann, G. A. \& Leibundgut, B. 1990, A\& A, 236, 9

Tegmark, M. 1999, ApJ, 514, L69

Thomsen, B., Baum, W. A., Hammergren, M. \& Worthey, G. 1997, ApJ, 483, L37

Tonry, J. L., Blakeslee, J. P., Ajhar, E. A. \& Dressler, A. 1997, ApJ, 475, 399

Tonry, J. L., Blakeslee, J. P., Ajhar, E. A. \& Dressler, A. 1999, astro-ph/9907062

Tonry, J. L. \& Franx, M. 1998, ApJ, 515, 512

Tonry, J. L. \& Schneider, D. P. 1988, AJ, 96, 807

Torres, G., Stefanik, R. P. \& Latham, D. W. 1997, ApJ, 474, 256

Tully, R. B. 1999, in Cosmological Parameters and the Evolution of the Universe (IAU

Symposium 183), ed. K. Sato (Kluwer, Dordrecht), p. 54

Tully, R. B. \& Fisher, J. R. 1977, A\& A, 54, 661

Tully, R. B. et al. 1998, ApJ, 115, 2264

Tyson, J. A. \& Fischer, P. 1995, ApJ, 446, L55

Udalski, A. 1998, Acta Astron., 48, 113

Udalski, A. et al. 1998a, Acta Astron., 48, 1

Udalski, A. et al. 1998b, ApJ, 509, L25

VandenBerg, D. A. \& Bridges, T. J. 1984, ApJ, 278, 679

VandenBerg, D. A., Bolte, M. \& Stetson, P. B. 1996, ARA\& A, 34, 461

de Vaucouleurs, G. 1981, in 10th Texas Symp. on Relativistic Astrophysics, ed. Ramaty, R. \& Jones, F. C., Ann. N.Y. Acad. Sci. 375, 90

Viana, P. T. P. \& Liddle, A. R. 1999, astro-ph/9902245 in Cosmological Constraints from X-Ray Clusters (to be published)

Weinberg, S. 1972, Gravitation and Cosmology (J. Wiley, New York)

White, D. A. \& Fabian, A. C. 1995, MNRAS, 273, 72

White, S. D. M., Efstathiou, G. \& Frenk, C. S. 1993a, MNRAS, 262, 1023

White, S. D. M., Navarro, J. F., Evrard, A. E. \& Frenk, C. S. 1993b, Nature, 366, 429

Whitelock, P. A., van Leeuwen, F. \& Feast. M. W. 1997, in Hipparcos Venice '97, ed.

B. Battrick (ESA, Noordwijk, 1997), p. 213

Willick, J. A. et al. 1997, ApJS, 109, 333

Yasuda, N., Fukugita, M. \& Okamura, S. 1997, ApJS, 108, 417

Zaldarriaga, M., Spergel, D. N. \& Seljak, U. 1997, ApJ, 488, 1

de Zeeuw, P. T. et al. 1997, in Hipparcos Venice' '97, ed. B. Battrick (ESA, Noordwijk, 1997), p. 495

Zaritsky, D., Smith, R., Frenk, C. \& White, S. D. M. 1997, ApJ, 478, 39 